\title{
Response of Saturn's auroral ionosphere to electron precipitation: Electron density, electron temperature, and electrical conductivity
}

\author{
Marina Galand, ${ }^{1,2}$ Luke Moore, ${ }^{2}$ Ingo Mueller-Wodarg, ${ }^{1,2}$ Michael Mendillo, ${ }^{2}$ \\ and Steve Miller ${ }^{3}$ \\ Received 29 December 2010; revised 30 April 2011; accepted 3 June 2011; published 10 September 2011.
}

[1] In the high-latitude regions of Saturn, the ionosphere is strongly coupled to the magnetosphere through the exchange of energy. The influx of energetic particles from Saturn's magnetosphere enhances the ionospheric densities and temperatures, affects the electrodynamical properties of the ionosphere, and contributes to the heating of the thermosphere. It is therefore critical to accurately model the energy deposition of these magnetospheric particles in the upper atmosphere in order to evaluate key ionospheric quantities of the coupled magnetosphere-ionosphere system. We present comprehensive results of ionospheric calculations in the auroral regions of Saturn using our Saturn Thermosphere-Ionosphere Model (STIM). We focus on solar minimum conditions during equinox. The atmospheric conditions are derived from the STIM 3-D General Circulation Model. The ionospheric component is self-consistently coupled to the solar and auroral energy deposition component. The precipitating electrons are assumed to have a

Maxwellian distribution in energy with a mean energy $E_{m}$ and an energy flux $\mathrm{Q}_{0}$. In the presence of hard electron precipitation $\left(1<E_{m} \leq 20 \mathrm{keV}\right)$ with $Q_{0}>0.04 \mathrm{~mW} \mathrm{~m}^{-2}$, the ionospheric conductances are found to be proportional to the square root of the energy flux, but the response of the ionosphere is not instantaneous and a time delay needs to be applied to $\mathrm{Q}_{0}$ when estimating the conductances. In the presence of soft electron precipitation $\left(E_{m}<500 \mathrm{eV}\right)$ with $Q_{0} \leq 0.2 \mathrm{~mW} \mathrm{~m}^{-2}$, the ionospheric conductances at noon are found to be primarily driven by the Sun. However, soft auroral electrons are efficient at increasing the ionospheric total electron content and at heating the thermal electron population.

Citation: Galand, M., L. Moore, I. Mueller-Wodarg, M. Mendillo, and S. Miller (2011), Response of Saturn's auroral ionosphere to electron precipitation: Electron density, electron temperature, and electrical conductivity, J. Geophys. Res., 116, A09306, doi:10.1029/2010JA016412.

\section{Introduction}

[2] Like all giant planets in the Solar System, Saturn is known to have an upper neutral atmosphere far hotter than what is expected from solar extreme ultraviolet (EUV) heating alone. While the measured exospheric temperatures on Saturn are close to $500 \mathrm{~K}$ over the full latitude range (R. Vervack and J. Moses, submitted manuscript, 2011), solar heating alone induces an exospheric temperature near $180 \mathrm{~K}$ [Yelle and Miller, 2004; Müller-Wodarg et al., 2006]. A major, additional source of energy originates in the highlatitude regions, where magnetospheric currents can deposit globally several tens of TW, more than 50 times the absorbed

\footnotetext{
${ }^{1}$ Space and Atmospheric Physics Group, Department of Physics, Imperia College London, London, UK.

${ }^{2}$ Center for Space Physics, Boston University, Boston, Massachusetts, USA.

${ }^{3}$ Atmospheric Physics Laboratory, Department of Physics and Astronomy, University College London, London, UK.

Copyright 2011 by the American Geophysical Union. 0148-0227/11/2010JA016412
}

solar EUV value, as thermal energy, primarily via Joule heating [Cowley et al., 2004a]. The assessment of Joule heating, plasma flows, and current system at high latitudes, all requires the knowledge of the ionospheric state, and in particular electrical conductances in the auroral regions [e.g., Cowley et al., 2004b, 2008].

[3] Energetic particles, primarily electrons, coming from the magnetosphere and affecting the ionospheric state induce spectacular auroral emissions. Such signatures have been extensively observed at Saturn in the EUV and the far ultraviolet (FUV) emitted by $\mathrm{H}$ and $\mathrm{H}_{2}$, in the infrared (IR) emitted by $\mathrm{H}_{3}^{+}$, in the kilometric radio range, and on the nightside in the visible (e.g., $\mathrm{H} \alpha$ ), as summarized in a recent review by Kurth et al. [2009]. Only an upper limit has been derived so far for auroral emissions in the X-rays [Hui et al., 2010].

[4] Several key features have emerged from these observations. The most intense electron precipitation occurs over a very narrow oval with a median latitudinal width varying from $1.5^{\circ}$ at dawn and dusk to $3.5^{\circ}$ at noon; though its poleward and equatorward boundaries are highly variable, the oval is typically located between $70^{\circ}$ and $85^{\circ}$ latitude [Badman et al., 2006]. An asymmetry between the southern and the 
northern hemispheres has also been recently reported by Nichols et al. [2009]. The brightness of the main oval varies with local time, with the most intense regions in the dawn and prenoon sectors [e.g., Cowley et al., 2004a; Grodent et al., 2005; Lamy et al., 2009]. There are however exceptions, as pointed out by Stallard et al. [2008a], who reported an unusual dusk-brightening event seen in the IR. The main auroral oval is also suggested to map to the upward current layer at the boundary between the open and closed magnetic field lines [e.g., Cowley et al., 2004a; Jackman and Cowley, 2006]. Though this finding is similar to what is seen at Earth, the processes involved are different [e.g., Clarke et al., 2005]. Instead of the terrestrial onset brightening observed near midnight during magnetic storms, Saturn's auroral emissions intensified in the dawn sector, as seen after shock-triggered events [e.g., Prangé et al., 2004]. Saturn's oval is also sensitive to the solar wind dynamic pressure, whose variations affect both the size of the main oval as well as its brightening [Clarke et al., 2005]. While at Jupiter the main oval is driven internally through the breaking from corotation [Cowley and Bunce, 2001], at Saturn such a process does not seem to be as efficient, in part due to the absence of a plasma source as strong as Io. Though weaker, this process however seems to be responsible for the auroral emissions, $25 \%$ as bright as the main oval, observed near $60^{\circ}$ latitude in the IR and most likely caused by mass loading from the torus of Enceladus [Stallard et al., 2008b, 2010]. Diffuse, weaker auroral emissions in the ultraviolet have also been observed equatorward of the main oval, centered on $67^{\circ} \mathrm{S}$ latitude over $7^{\circ}$ and associated with softer electrons $(<\mathrm{keV})$ than those responsible for the main oval $(>\mathrm{keV})$ [Pryor et al., 2009; Grodent et al., 2010].

[5] While numerous modeling studies of the deposition of auroral energetic particles and the consecutive response of the ionosphere have been undertaken at Jupiter [e.g., Waite et al., 1983; Régo et al., 1994; Perry et al., 1999; Grodent et al., 2001; Millward et al., 2002; Hiraki and Tao, 2008; Menager et al., 2010], only a few dedicated studies have yet been published at Saturn. There are studies from Waite [1981] and Gérard and Singh [1982], which are based on nonrealistic thermospheric conditions, as known now, and studies focusing on auroral emissions with the electron energy deposition modeled in a pure $\mathrm{H}_{2}$ atmosphere [e.g., Gustin et al., 2009].

[6] We have undertaken a comprehensive assessment of plasma processes in the auroral ionosphere of Saturn. For that purpose, we have used our Saturn Thermosphere-Ionosphere Model (STIM), whose components are described in section 2. In section 3, we describe the input parameters used for the simulations. We next discuss the thermospheric conditions and pressure-altitude conversion in the auroral regions (section 4). Two auroral electron cases, soft and hard, are selected and compared in terms of energy deposition (section 5) and in terms of ionospheric response (section 6). A third case which includes only solar illumination is used as a reference. Results are discussed in section 7.

\section{Model}

[7] In order to assess the ionospheric conditions in the auroral regions of Saturn, we have used our STIM model to which we have recently added a self-consistent calculation of the auroral forcing. The 3-D thermosphere-ionosphere module solves the continuity, momentum, and energy equations for the atmospheric neutral species [Müller-Wodarg et al., 2006] and for the thermal ion species [Moore et al., 2004, 2008, 2009]. The thermosphere and ionosphere are fully coupled dynamically through collisions between ions and neutrals. The high-latitude forcing is fully self-consistent. The Joule heating is explicitly calculated from the electrical conductances affected by solar illumination and auroral electron precipitation. The $3-\mathrm{D}$ model resolution is $2^{\circ}$ in latitude and $10^{\circ}$ in longitude.

[8] The 1D energy deposition module, validated for auroral electrons at a giant planet in Appendix A, describes the absorption of solar photons by solving the Lambert-Beer Law and the transport, energy degradation, and angular redistribution of suprathermal electrons by solving the Boltzmann equation [Moore et al., 2008, 2009; Galand et al., 2009]. The suprathermal electron population includes the photoelectrons produced through photoionization, the auroral electrons precipitating from Saturn's magnetosphere, and the secondary electrons produced via ionization of the neutral atmospheric species by any suprathermal electrons. The coupling between the ionospheric and the energy deposition modules is based on the exchange of thermal electron density and temperature, ion production rates, and thermal electron heating rates [Moore et al., 2008].

\section{Input Parameters Used}

[9] All the simulations whose results are presented in sections 4,5 , and 6 were performed for equinox and solar minimum conditions, which are representative of the conditions encountered by the Cassini spacecraft at Saturn in 2009. The latitude is set to $78^{\circ} \mathrm{S}$. This corresponds to a magnetic dip angle of $82^{\circ}$ and a solar zenith angle (SZA) of $78^{\circ}$ at noon during equinox.

[10] The solar flux at the top of the atmosphere is based on measurements by the Thermosphere Ionosphere Mesosphere Energetics and Dynamics (TIMED)/Solar EUV Experiment (SEE) extrapolated to Saturn for May 15, 2008 [Woods et al., 2000, 2005; Woods, 2008; Galand et al., 2009]. It is associated with an ionizing energy flux in the soft X-ray and EUV range (up to $98 \mathrm{~nm}$ corresponding to the ionization threshold of $\mathrm{CH}_{4}$ ) of $3.06 \times 10^{-2} \mathrm{~mW} \mathrm{~m}^{-2}$. For a solar zenith angle of $78^{\circ}$ (noon), $22 \%$ of this radiation is absorbed along the local vertical. This modest percentage is due to the large SZA considered and, therefore, the strong atmospheric absorption of the solar flux before reaching the atmospheric vertical column of interest.

[11] In order to assess the ionospheric response to electron precipitation, two cases are considered, one associated with hard electrons with a mean energy, $\mathrm{E}_{m}$, of $10 \mathrm{keV}$ and one associated with soft electrons with $\mathrm{E}_{m}$ of $500 \mathrm{eV}$. The energy distribution of the incident auroral electrons is assumed to be Maxwellian. The hard energy value is consistent with those observed in the main auroral oval and derived from the analysis of ultraviolet auroral observations from Voyager/ ultraviolet spectrometer (UVS) (10 keV and less [Sandel et al., 1982]) and from Hubble Space Telescope (HST) (12 keV \pm $3 \mathrm{keV}$ [Gérard et al., 2004], 5-30 keV [Gérard et al., 2009], 10-18 keV [Gustin et al., 2009]). It is also consistent with Cassini/CAPS electron observations providing mean ener- 

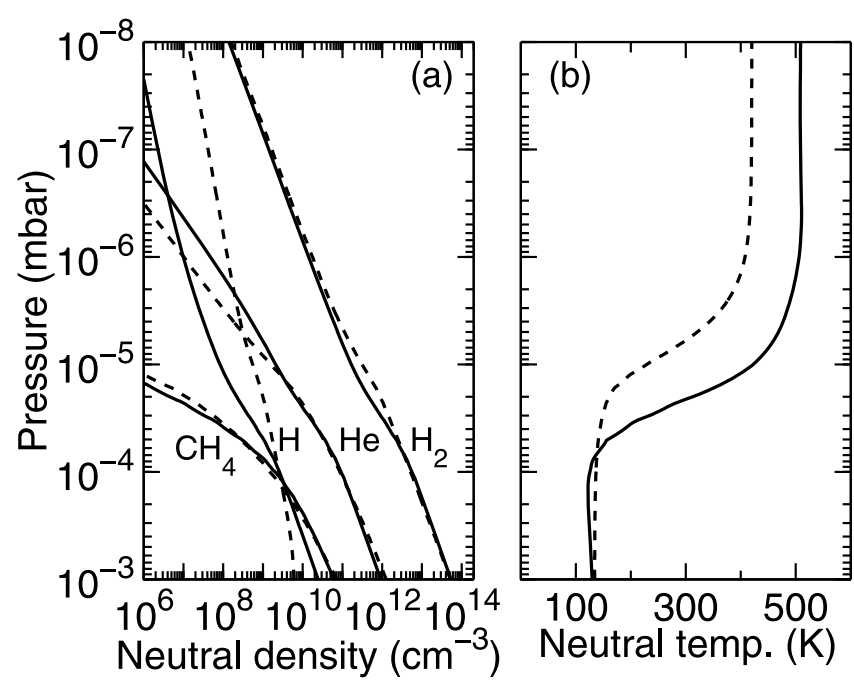

Figure 1. Neutral atmospheric (a) density and (b) temperature profiles in altitude resulting from the 3-D neutral STIM model at $78^{\circ} \mathrm{S}$ latitude at equinox $\left(\mathrm{T}_{\text {exo }}=510 \mathrm{~K}\right)$ (solid lines). The reference profiles $\left(\mathrm{T}_{\text {exo }}=420 \mathrm{~K}\right)$ derived by Moses et al. [2000] are shown as dashed lines.

gies in the $6-9 \mathrm{keV}$ range and associated with a source region of Saturn kilometric radiation (SKR) on the nightside [Lamy et al., 2010]. These latter values provide however a lower limit as the electrons most likely undergo acceleration before reaching the ionosphere. The mean energy value for the soft electron precipitation is consistent with diffuse aurora observed by Cassini/UVIS and extending equatorward of the main oval with energies estimated to range between 400 and $800 \mathrm{eV}$ [Pryor et al., 2009]. The softness of the precipitation is confirmed by the analysis of HST/STIS FUV $\mathrm{H}_{2}$ emissions which did not suffer any significant methane absorption [Grodent et al., 2010].

[12] The energy flux of the precipitating electrons, $\mathrm{Q}_{0}$, defined over the downward hemisphere is given in $\mathrm{mW} \mathrm{m}^{-2}$ by:

$$
Q_{0}=2 \pi \int_{E_{\min }}^{E_{\max }} \int_{-1}^{0} I_{e}\left(z_{t o p}, E, \mu\right) E \mu d E d \mu
$$

where $I_{e}$ is the suprathermal electron intensity in $\mathrm{cm}^{-2} \mathrm{~s}^{-1}$ $\mathrm{erg}^{-1} \mathrm{sr}^{-1}, z_{\text {top }}$, the altitude of the top of the atmosphere, here taken equal to $2650 \mathrm{~km}\left(4 \times 10^{-9} \mathrm{mbar}\right), E$, the energy of the suprathermal electrons, and $\mu$, the cosine of their pitch angle. For auroral electron precipitation assumed to be isotropic over the downward hemisphere, which is the case here, the right side of relation (1) reduces to $\pi \int_{E_{\min }}^{E_{\max }} I_{e}\left(z_{\text {top }}, E\right)$ $E d E . \mathrm{Q}_{0}$ is assumed to be constant, equal to $0.2 \mathrm{~mW} \mathrm{~m}^{-2}$, over all local times. This corresponds to twice the total solar incident energy flux. The value chosen for $\mathrm{Q}_{0}$ is consistent with values in the dusk, afternoon, and premidnight sector derived from UV analysis of auroral images [e.g., Cowley et al., 2004a].

[13] Overall it is representative of very quiet conditions in the auroral oval. Indeed, typical auroral brightnesses observed in the $\mathrm{H}_{2}$ Lyman and Werner bands range from a few $\mathrm{kR}$ to 15 kR during quiet conditions [e.g., Gérard et al., 2004;
Grodent et al., 2005; Gustin et al., 2009]. Assuming that auroral electrons with $\mathrm{Q}_{0}$ of $1 \mathrm{~mW} \mathrm{~m} \mathrm{~m}^{-2}$ generate a $10 \mathrm{kR} \mathrm{H}_{2}$ brightness in the Lyman and Werner bands [e.g., Gérard and Singh, 1982; Waite et al., 1983; Grodent et al., 2001], these values translate to an incident energy flux $\mathrm{Q}_{0}$ between a few tenths of $\mathrm{mW} \mathrm{m}^{-2}$ to $1.5 \mathrm{~mW} \mathrm{~m}^{-2}$.

[14] All results presented in sections 5 and 6 have been obtained from runs in which the ionospheric and the energy deposition modules were self-consistently coupled over a full Saturn day (see section 2). The default time step of the coupling is 10 Saturn minutes, that is, 4 Earth min $26 \mathrm{~s}$ (with 1 Saturn minute defined as 1 minute of a $24 \mathrm{~h}$ Saturn day and corresponding to 26.6 "Earth" seconds for a rotation period for Saturn of $10 \mathrm{~h} 39 \mathrm{~min}( \pm 8 \mathrm{~min}$ ) [Anderson and Schubert, 2007; Gurnett et al., 2005; Zarka et al., 2007; Helled et al., 2009; Read et al., 2009]). The magnetic field configuration is taken from the Saturn Pioneer Voyager model [Davis and Smith, 1990], which provides a sufficient accuracy for the current purpose. It should be noted that no water is considered in the ionospheric auroral simulations. At low latitudes, water plays an important role where it provides a loss mechanism for the ionosphere [e.g., Connerney and Waite, 1984; Moses and Bass, 2000; Moore and Mendillo, 2007; Moore et al., 2006, 2010].

[15] The effective reaction rate $\mathrm{k}_{1}^{*}$ for charge exchange between $\mathrm{H}^{+}$and vibrationally excited $\mathrm{H}_{2}$ is given by:

$$
k_{1}^{*}=k_{1} \frac{\left[H_{2}(\nu \geq 4)\right]}{\left[H_{2}\right]} \quad\left[\mathrm{cm}^{3} \mathrm{~s}^{-1}\right]
$$

with $\mathrm{k}_{1}$, the actual reaction rate taken to be $10^{-9} \mathrm{~cm}^{3} \mathrm{~s}^{-1}$ [Huestis, 2008]. The fraction of vibrationnally excited $\mathrm{H}_{2}$ density, $\left[\mathrm{H}_{2}(\nu \geq 4)\right] /\left[\mathrm{H}_{2}\right]$, is assumed to be twice that proposed by Moses and Bass [2000], as in the auroral region a larger fraction of excited $\mathrm{H}_{2}$ is expected, as discussed in section 7.5. The interpolation is done on the pressure grid. The effective $\mathrm{H}_{2}$ vibrational reaction rate $\mathrm{k}_{1}^{*}$ is equal to $9.5 \times$ $10^{-20} \mathrm{~cm}^{3} \mathrm{~s}^{-1}$ at $600 \mathrm{~km}$ to $7.33 \times 10^{-14} \mathrm{~cm}^{3} \mathrm{~s}^{-1}$ at $2700 \mathrm{~km}$.

[16] The thermospheric density and temperature profiles used as input of the ionospheric and energy deposition modules are derived from the full 3-D STIM model for each local time considered. They are discussed in section 4 .

\section{Thermospheric Conditions at Saturn}

[17] In the 3-D STIM model from which the thermospheric conditions are extracted, the auroral oval is represented by a sharp gaussian, covering primarily one $2^{\circ}$ bin in latitude, centered around $78^{\circ}$ in the northern and southern hemispheres, in agreement with the location of the openclose magnetic field line boundary [Cowley et al., 2004a].

[18] The energy distribution of the auroral electrons is a Maxwellian distribution with a mean energy of $10 \mathrm{keV}$. The external magnetospheric electric field assumed is based on the one proposed by Cowley et al. [2004a]. It is pointing equatorward and is assumed to be constant with local time and longitude.

[19] The thermospheric densities and temperature extracted at a latitude of $78^{\circ} \mathrm{S}$ at $12 \mathrm{LT}$ at equinox during solar minimum conditions (see section 3) are presented in Figure 1. Though the quantities are plotted for noon, no significant 


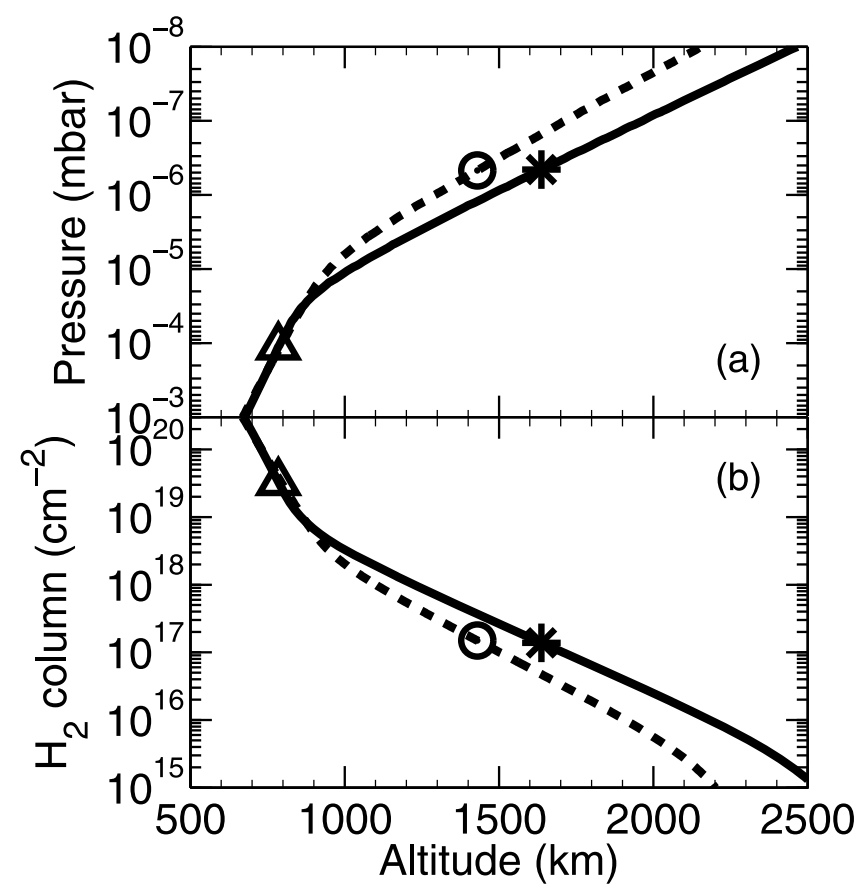

Figure 2. (a) Pressure versus altitude derived from the 3-D STIM atmospheric profile $\left(\mathrm{T}_{\text {exo }}=510 \mathrm{~K}\right)$ (solid line) and from the reference profile by Moses et al. [2000] $\left(\mathrm{T}_{\text {exo }}=\right.$ $420 \mathrm{~K}$ ) (dashed line). (b) Same as Figure 2a but for the $\mathrm{H}_{2}$ column density above. The markers correspond to the pressure and $\mathrm{H}_{2}$ column density at the altitude of maximum energy deposition for $E_{m}=10 \mathrm{keV}$ (triangle) using the STIM atmosphere and for $E_{m}=500 \mathrm{eV}$ using the $78^{\circ} \mathrm{S}$ STIM atmosphere (star) and reference atmosphere (circle). For visibility reasons, the case of $10 \mathrm{keV}$ using the reference atmosphere has not been plotted as it overlaps with the $10 \mathrm{keV}$ case using the STIM atmosphere. The transport of auroral electrons and the column density valid along the path were calculated for the dip angle at $78^{\circ} \mathrm{S}$, i.e., $82^{\circ}$. The reference altitude is taken to be the 1 bar level, and the top of the atmosphere is taken at a pressure of $4.4 \times 10^{-9}$ mbar.

local time dependence is found, which is explained by the fast rotation of the planet. The exospheric temperature, $\mathrm{T}_{\text {exo }}$, is strongly correlated with the amount of Joule heating present in the high-latitude regions. The electron energy flux and convection electric field, which drive the Joule heating, are chosen such that the derived value for $\mathrm{T}_{\text {exo }}$ is in agreement with observations from UV occultations.

[20] Recently, Vervack and Moses (manuscript in preparation, 2011) reanalyzed the Voyager UVS occultations and found observed values near $500 \mathrm{~K}$ for $\mathrm{T}_{\text {exo }}$ over the full latitude range. The value of $510 \mathrm{~K}$ derived from the present STIM run (see Figure 1b) is consistent with the result of this latest analysis. It was obtained by reducing the electric field of Cowley et al. [2004a] by $60 \%$ (giving a peak electric field strength of $4 \mathrm{mV} \mathrm{m}^{-1}$ ) and by assuming an energy flux of the auroral electrons equal to $0.2 \mathrm{~mW} \mathrm{~m}^{-2}$. Further simulations have shown that any combination of auroral electron fluxes and electric field strength that yields the same exospheric temperatures produce similar thermospheric density profiles. For instance, doubling the energy flux and reducing consequently the electric field for reaching an exospheric temperature of $510 \mathrm{~K}$ change the mixing ratios for $\mathrm{H}$ and $\mathrm{He}$ by less than $1 \%$. The choice of electric field affects neutral dynamics, via ion acceleration and ion-neutral drag, and this in turn affects vertical winds and composition, but the ultimate effect on mixing ratios is minor. The derived atmospheric profiles presented in Figure 1 thus represent a valid choice for assessing the auroral energy deposition.

[21] For reference, the midlatitude atmospheric model of Moses et al. [2000], widely used by the community at high latitudes [e.g., Gérard et al., 2004; Gustin et al., 2009] is shown as dashed lines in Figure 1. The associated exospheric temperature of $420 \mathrm{~K}$ was derived by Smith et al. [1983] from the analysis of the Voyager 2 UVS solar ingress occultation. It should be noted that the recent reanalysis of this occultation by Vervack and Moses (manuscript in preparation, 2011) yields a revised exospheric temperature value of $480 \mathrm{~K}$. Plotted as a function of pressure, the $\mathrm{H}_{2}$ density obtained for $\mathrm{T}_{\text {exo }}=510 \mathrm{~K}$ is very close to the $420 \mathrm{~K}$ reference model. The same applies to the minor species $\mathrm{He}$ and $\mathrm{CH}_{4}$ at high pressures above $3 \times 10^{-5}$ mbar, keeping in mind that the mixing ratios assumed for these species at the lower boundary in the STIM model are those from Moses et al. [2000]. At lower pressure, large differences in the $\mathrm{H}$ and $\mathrm{He}$ densities are clearly apparent between the STIM profiles and the reference profiles. Such differences are primarily due to global dynamics.

[22] Because of the difference in temperature profiles between the $510 \mathrm{~K} \mathrm{STIM}$ (solid line) and the $420 \mathrm{~K}$ reference (dashed line) atmospheric models (see Figure 1b), the conversion from pressure to altitude is similar at pressures larger than $2 \times 10^{-5}$ mbar, but significantly different at pressures smaller than $2 \times 10^{-5}$ mbar, as illustrated in Figure $2 \mathrm{a}$. For instance, on the one hand, $10 \mathrm{keV}$ auroral electrons penetrate deeply into the atmosphere to an altitude of $790 \mathrm{~km}$ (triangle in Figure 2a) corresponding to an $\mathrm{H}_{2}$ column density of $3 \times 10^{19} \mathrm{~cm}^{-2}$ (triangle in Figure $2 \mathrm{~b}$ ) and have a penetration altitude largely insensitive to the atmospheric models. On the other hand, for $500 \mathrm{eV}$ electrons there is a difference of $208 \mathrm{~km}$ between the high-latitude STIM (stars) and the midlatitude reference (circles) models. Therefore, the conversion between the penetration altitude of auroral electrons and the mean energy of the auroral electrons using alternative atmospheric models needs to be taken with caution when focusing on soft electron precipitation.

\section{Energy Deposition of Auroral Electrons}

[23] The energy sources considered include hard and soft auroral electron precipitations and solar radiation under solar minimum conditions (see section 3 ). The energy flux carried by photoelectrons $\left(3.8 \times 10^{-3} \mathrm{~mW} \mathrm{m^{-2 }}\right.$ for a SZA of $\left.78^{\circ}\right)$ represents $56 \%$ of the total ionizing solar radiation absorbed in the atmosphere $\left(6.8 \times 10^{-3} \mathrm{~mW} \mathrm{~m}^{-2}\right.$ for a SZA of $\left.78^{\circ}\right)$. This value is consistent with the value of $47 \%$ found at Jupiter [Waite et al., 1983]. The rest of the absorbed solar energy is used for ionizing atmospheric species and, to a much lesser extent, exciting and dissociating molecules.

[24] Figures $3 a$ and $3 b$ show the profiles of the electron production rate and the electron heating rate at noon $\left(78^{\circ} \mathrm{SZA}\right)$.

[25] The rates for the soft and the hard cases are shown with solid lines and dashed lines, respectively, and include both the auroral and solar contribution through electron 

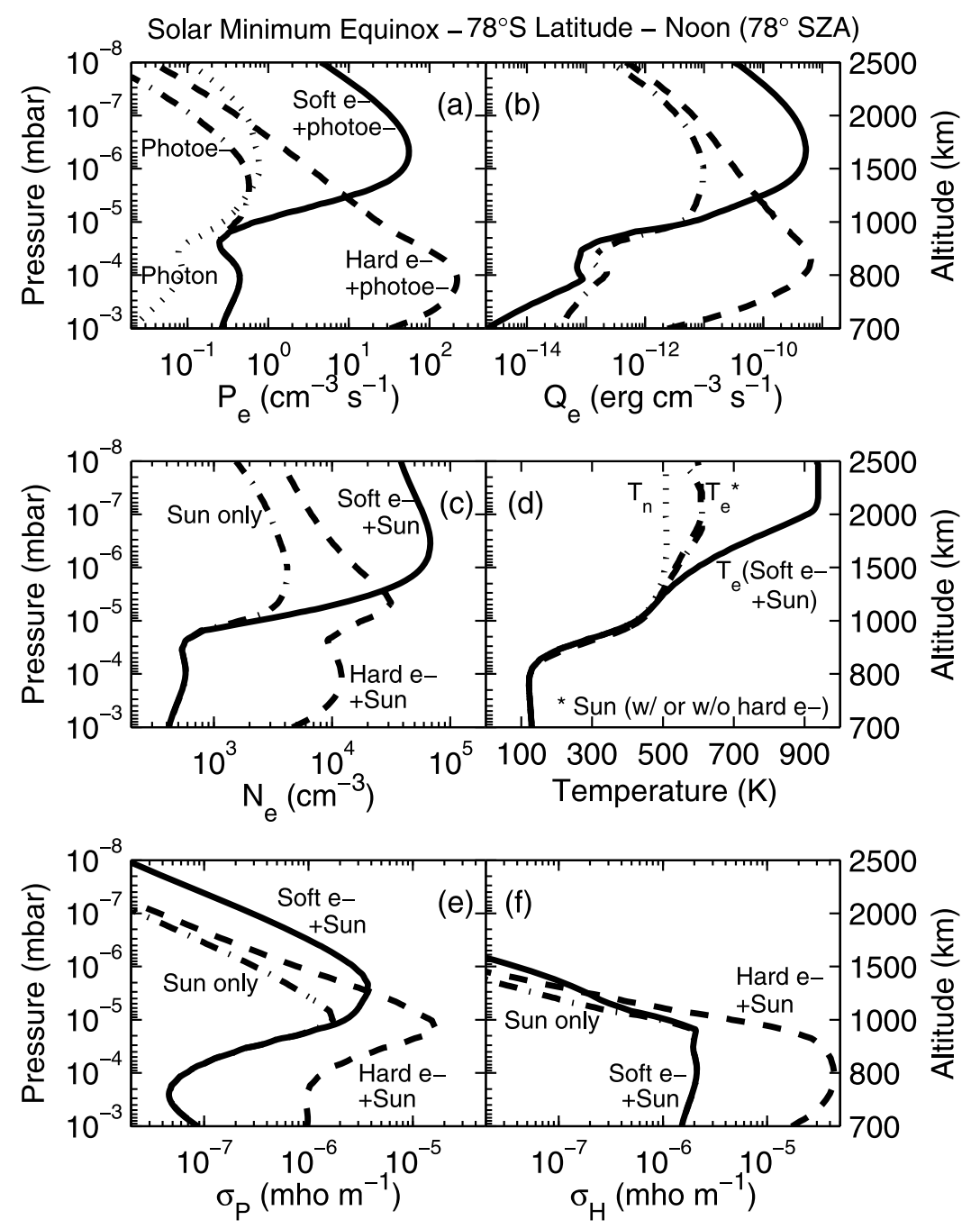

Figure 3. (a) Secondary electron production rates from STIM calculations at $78^{\circ} \mathrm{S}$ latitude, at noon $\left(\mathrm{SZA}=78^{\circ}\right)$, at equinox, during solar minimum, for the soft electron case [auroral electrons $\left(E_{m}=\right.$ $500 \mathrm{eV}, Q_{0}=0.2 \mathrm{~mW} \mathrm{~m}^{-2}$ ) + photoelectrons] (solid line), the hard electron case [auroral electrons $\left(E_{m}=10 \mathrm{keV}, Q_{0}=0.2 \mathrm{~mW} \mathrm{~m}^{-2}\right)+$ photoelectrons] (dashed line), and solar illumination alone (photoelectrons) (dash-dotted line). The energy distribution of the incident auroral electrons is a Maxwellian. The primary electron production due to photoionization by solar photons is plotted with dotted line. (b) Same as Figure 3a but for the thermal electron heating rate. (c) Same as Figure 3a but for electron density. The dash-dotted line represents the electron density derived from solar illumination alone taking into account both photo- and electron-impact ionization. (d) Same as Figure 3a but for electron temperature. The neutral temperature, same as shown in Figure $1 \mathrm{~b}$, is plotted as dotted line for reference. (e) Same as Figure 3a but for Pedersen conductivities. (f) Same as Figure 3a but for Hall conductivities. The vertical axis on the right provides an approximate altitude value of the pressures given on the vertical axis on the left using the thermospheric conditions from Figure 1.

impact. The rates produced by photoelectrons alone are shown as dash-dotted line. The primary electron production rate associated with photoionization (dotted line) is also shown in Figure 3a for reference. The thermospheric conditions used are described in section 4 . The altitude of deposition of $500 \mathrm{eV}$ electrons is near $1600 \mathrm{~km}\left(5 \times 10^{-7} \mathrm{mbar}\right)$, close to the region of deposition of solar HeII $(30.4 \mathrm{~nm})$ which drives the main peak of the primary electron production rate. When present, soft auroral electrons are the dominant ionization and thermal electron heating source in this region. Photoelectrons and their secondaries produce a secondary peak in electron production at lower altitudes, near $800 \mathrm{~km}\left(8 \times 10^{-5} \mathrm{mbar}\right)$, where soft X-rays deposit their energy [Galand et al., 2009]. This peak is not affected by the presence of soft auroral electrons. However, hard auroral electrons, when present, also deposit their energy near $800 \mathrm{~km}\left(8 \times 10^{-5}\right.$ mbar $)$ and become the dominant source of ionization and thermal electron heating in this region.

[26] Suprathermal electrons lose energy through collisions with atmospheric neutrals yielding ionization, excitation, and dissociation. At low energies, typically below a few eV, 
Coulomb collisions transferring energy from suprathermal electrons to thermal, ionospheric electrons become dominant. This process depends on the thermal electron density and temperature. Most energy of the auroral electrons is lost through collisions with neutrals $(79 \%$ for $500 \mathrm{eV}$ electrons and $89 \%$ for $10 \mathrm{keV}$ electrons) with the remaining lost through collisions with thermal electrons (16\% for $500 \mathrm{eV}$ electrons and $7 \%$ for $10 \mathrm{keV}$ electrons) and lost at the top of the atmosphere as a result of collisional scattering (5\% for $500 \mathrm{eV}$ electrons and $4 \%$ for $10 \mathrm{keV}$ electrons). In addition, from the energy deposited in the neutrals, more than $90 \%$ is lost through collisions with $\mathrm{H}_{2}$ and about $50 \%$ of the total energy is used for ionizing $\mathrm{H}_{2}$ and producing $\mathrm{H}_{2}^{+}$, the major ion produced through electron impact. The percentages provided above are for "pure" auroral electron precipitation, in the absence of solar illumination. As photoelectrons are less energetic than the auroral electrons considered here, they deposit slightly less energy through collisions with neutrals $(76 \%)$ and more through collisions with the thermal, ionospheric electrons (21\%) than auroral electrons do. This is illustrated in Figures $3 \mathrm{a}$ and $3 \mathrm{~b}$ through the relative importance of the peak magnitude of the ionization and thermal electron heating rates. For instance, the peak electron production rate ratio between the soft electron and the pure photoelectron cases is larger than the peak electron heating rate ratio between the same two cases.

\section{Ionospheric Response}

\subsection{Electron and Ion Densities}

[27] Figure 3c shows the profiles of the electron density for the soft electron (solid line) and hard electron (dashed line) cases at noon ( $\left.78^{\circ} \mathrm{SZA}\right)$. The solar contribution is included in the auroral profiles. For reference, the electron density profile produced under solar illumination alone is shown with the dash-dotted line.

[28] In all shown electron density profiles the main peak is located above the homopause level ( $\approx 1000 \mathrm{~km}$ or $\left.10^{-5} \mathrm{mbar}\right)$. For the case of solar illumination alone the principal ion at the peak is $\mathrm{H}_{3}^{+}$, while in the cases with electron precipitation it is $\mathrm{H}^{+}$. As the auroral electrons are assumed to precipitate at a constant rate at all local times, the $\mathrm{H}^{+}$density, which does not show any significant diurnal variation above $1500 \mathrm{~km}\left(9 \times 10^{-7} \mathrm{mbar}\right)$ due to its long chemical lifetime, drives the main electron density peak. In the absence of auroral electron precipitation, the ionization source only occurs during daylight, that is, under solar illumination. At noon, the main peak is associated with $\mathrm{H}_{3}^{+}$. After sunrise, $\mathrm{H}_{3}^{+}$ builds up faster than $\mathrm{H}^{+}$, as the production of $\mathrm{H}_{3}^{+}$from $\mathrm{H}_{2}^{+}$, the main ion produced through photoionization and impact ionization due to photoelectrons and their secondaries, is faster than the production of $\mathrm{H}^{+}$from $\mathrm{H}_{2}^{+}$[e.g., Moore et al., 2004; Galand et al., 2009]. The presence of soft electrons increases the main peak electron density by a factor of 16 , while the presence of hard electrons, which deposit most of their energy lower down (see Figure 3a) increases it by a factor of 7.5. Near the homopause, $\mathrm{H}_{3}^{+}$is the dominant ion species for all three cases due to the loss of $\mathrm{H}^{+}$through charge-exchange reaction with hydrocarbons, which is twice as fast as that of $\mathrm{H}_{3}^{+}$.

[29] Below the homopause, the main loss process of the $\mathrm{H}^{+}$and $\mathrm{H}_{3}^{+}$ions is charge-exchange with hydrocarbon neu- trals. As a result, the secondary peak seen in the electron density profiles below the homopause, produced by photoelectrons from soft $\mathrm{X}$-rays and hard electron precipitation (see Figure 3a), is associated with hydrocarbon ions. The presence of hard electron precipitation increases the secondary electron density peak by a factor 20 .

\subsection{Electron and Ion Temperatures}

[30] Figure 3d shows the electron temperature profiles produced by soft electrons (solid line) and hard electrons (dashed line) at noon ( $\left.78^{\circ} \mathrm{SZA}\right)$. The solar contribution is included in the auroral profiles. For reference, the electron temperature profile under solar illumination alone is shown as dash-dotted line. Energy is transferred from suprathermal electrons to ionospheric, thermal electrons, which yields an increase of the electron temperature above the neutral temperature (dotted line) to $610 \mathrm{~K}$ at $2500 \mathrm{~km}\left(9 \times 10^{-9} \mathrm{mbar}\right)$ [Moore et al., 2008]. The presence of soft electrons, which are efficient to heat at high altitudes above $1250 \mathrm{~km}(3 \times$ $10^{-6}$ mbar) (see Figure $3 \mathrm{~b}$ ), increases the electron temperature to $940 \mathrm{~K}$ at $2500 \mathrm{~km}$. Hard electrons also generate a large heating rate but at lower altitudes below $1250 \mathrm{~km}$ (see Figure $3 \mathrm{~b}$ ), in a region of large neutral densities, and thus efficient cooling. As a result, in this region the electron temperature is equal to the neutral temperature (see Figure $3 \mathrm{~d}$ ). Over the whole altitude range considered, the $\mathrm{H}_{3}^{+}$temperature (not shown) is less than 3\% higher than the neutral temperature for the solar and hard electron cases and less than $38 \%$ higher for the soft electron case.

\subsection{Electrical Conductivities and Conductances}

[31] The current density is the sum over the various charged species of their charge density multiplied by their velocity. To a good approximation, the velocity can be obtained by balancing the Lorentz force and the frictional force caused by collisions with neutrals [e.g., Richmond, 1995]. The resultant conductivity expressions are

$$
\begin{gathered}
\sigma_{P}=\sum_{i} \sum_{n} \frac{N_{i} e}{B}\left(\frac{\nu_{e n \perp} \Omega_{e}}{\nu_{e n \perp}^{2}+\Omega_{e}^{2}}+\frac{\nu_{i n} \Omega_{i}}{\nu_{i n}^{2}+\Omega_{i}^{2}}\right) \\
\sigma_{H}=\sum_{i} \sum_{n} \frac{N_{i} e}{B}\left(\frac{\Omega_{e}^{2}}{\nu_{e n \perp}^{2}+\Omega_{e}^{2}}-\frac{\Omega_{i}^{2}}{\nu_{i n}^{2}+\Omega_{i}^{2}}\right),
\end{gathered}
$$

where $\sigma_{P}$ and $\sigma_{H}$ are Pedersen and Hall conductivities, respectively, in mho $\mathrm{m}^{-1}, N_{i}$ is the density of the ion species $i\left(\mathrm{H}^{+}, \mathrm{He}^{+}, \mathrm{H}_{2}^{+}, \mathrm{H}_{3}^{+}\right.$, hydrocarbon ions) in $\mathrm{m}^{-3}, e$ is the magnitude of the electron charge in Coulomb, and $B$ is the magnitude of the magnetic field in Tesla. Here $\Omega_{e}$ and $\Omega_{i}$ are the angular gyrofrequencies in $\mathrm{s}^{-1}$ of the electrons and the ion species $i$, respectively: $\Omega=e B / m$, where $m$ is the mass of the particle (electron or ion) in kilograms. Here $\nu_{e n \perp}$ represents the effective collision frequency between the electrons and the neutral species $n\left(\mathrm{H}_{2}, \mathrm{H}, \mathrm{He}, \mathrm{CH}_{4}\right)$ for motions perpendicular to $\mathbf{B}$, and $\nu_{i n}$ is the (isotropic) collision frequency between the ion species $i$ and the neutral species $n$ [Banks and Kockarts, 1973].

[32] The magnetic field is taken from the Saturn Pioneer Voyager model [Davis and Smith, 1990]. The collision frequencies between ion species and neutral species are from 


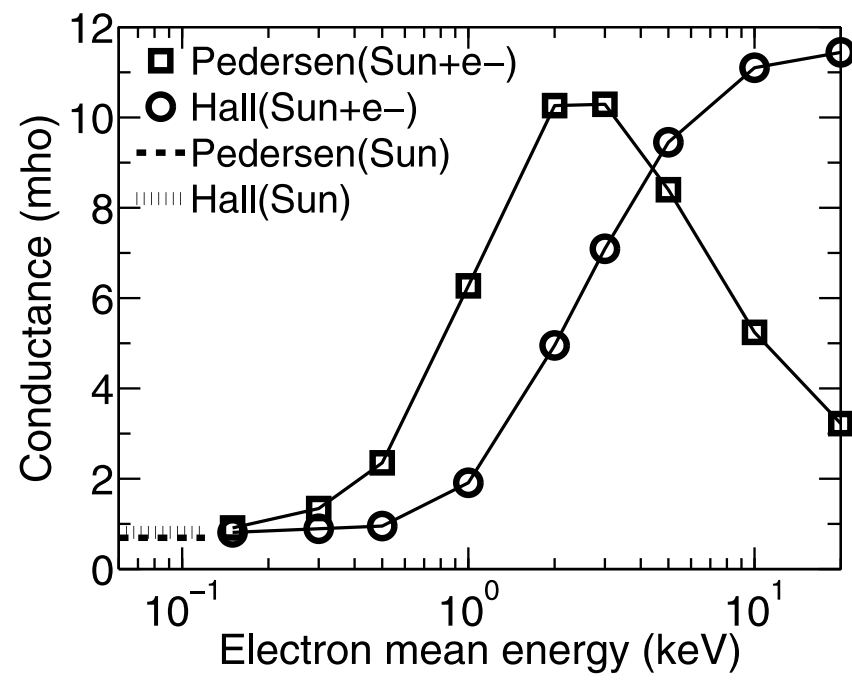

Figure 4. Pedersen (squares) and Hall (circles) ionospheric conductances as a function of the mean energy of the incident auroral electrons, $E_{m}$, under solar illumination at noon (SZA of $78^{\circ}$ ). The distribution of the incident electrons is assumed to be Maxwellian with an energy flux, $Q_{0}$, of $0.2 \mathrm{~mW} \mathrm{~m}^{-2}$. The Pedersen and Hall ionospheric conductances obtained for solar illumination alone (no auroral particle precipitation) are shown with horizontal, dotted and dashed lines, respectively.

Schunk and Nagy [2009]. The atmospheric densities and temperatures are from the STIM model. The electrical Pedersen and Hall conductances, $\Sigma_{P}$ and $\Sigma_{H}$, are obtained by integrating over height the ionospheric conductivities, $\sigma_{P}$ and $\sigma_{H}$, respectively.

\subsubsection{Dependence of the Electrical Conductances on the Auroral Electron Energy}

[33] Figures $3 \mathrm{e}$ and $3 \mathrm{f}$ show the ionospheric Pedersen and Hall conductivity profiles, respectively, for the soft electron (solid line) and the hard electron (dashed line) cases at noon $\left(78^{\circ} \mathrm{SZA}\right)$. The solar contribution is included in the auroral profiles. For reference, the conductivity profiles induced under solar illumination alone, in the absence of auroral particle precipitation, are shown with dash-dotted lines. The peak of the Pedersen conductivities is located near or above the homopause where it is driven by thermal ions. Due to the small ratio between $\nu_{e n}$ and $\Omega_{e}$, electrons contribute little to the Pedersen conductivity, similar to the terrestrial case, at and above E layer altitudes [Kelley, 1989; Moore et al., 2010]. The peak of the Hall conductivities is located below the homopause where it is driven by thermal electrons. Ion composition influences conductivities, as reported by Hinson et al. [1998] and Moore et al. [2010]. The presence of soft electron precipitation modestly increases the Pedersen conductivity and has no significant contribution to the Hall conductivity, while hard electron precipitation significantly contributes to the conductivities, especially near and below the homopause.

[34] In Figure 4, the ionospheric Pedersen conductance $\Sigma_{P}$ (squares) and Hall conductance $\Sigma_{H}$ (circles) are plotted as a function of the mean energy of the incident electrons. The energy dependence is different between Pedersen and
Hall conductances, as their conductivities do not peak in the same altitude region (see Figures $3 e$ and $3 f$ ). Auroral electrons with incident mean energy in the $2-3 \mathrm{keV}$ range deposit their energy near the homopause, where the Pedersen conductivity peaks. As a result, the Pedersen conductance reaches a maximum in the $2-3 \mathrm{keV}$ incident energy range. The Hall conductivity peaks well below the homopause. As a consequence, the Hall conductance is increasing over the whole electron mean energy range considered here spreading from $150 \mathrm{eV}$ to $20 \mathrm{keV}$. At very low electron mean energy, the auroral deposition peak altitude is located at altitudes higher than the solar deposition peak altitude, in a region less efficient for generating conductivities. As a result, the Pedersen and Hall conductances converge towards the solar values equal to $0.7 \mathrm{mho}$ and $0.8 \mathrm{mho}$, respectively. At $150 \mathrm{eV}$, the Pedersen conductance is within $30 \%$ of the solar value, while the Hall conductance whose conductivity peaks lower than the Pedersen conductivities, has already reached the solar value.

\subsubsection{Dependence of the Electrical Conductances on the Auroral Electron Flux}

[35] In all the simulations presented so far in this paper, we have assumed a constant energy flux, $Q_{0}$, for the incident auroral electrons. However, the dependence in local time (LT) of the brightness of the auroral emissions, and hence of the energy flux of the incident auroral electrons, has been widely reported [e.g., Cowley et al., 2004a; Grodent et al., 2005; Lamy et al., 2009]. We have therefore performed an additional simulation in which the energy flux of the auroral electrons, $Q_{0}$, varies with LT, as shown in Figure 5a (solid line). The relative LT variability of the energy flux is based on the LT variability of the UV brightness inferred from the analysis of HST/UV images using $5^{\circ}$ longitudinal bins within $\left[-70^{\circ},-80^{\circ}\right]$ latitudinal range where most of the aurora lies [Lamy et al., 2009]. A smoothing process has been applied to the profile, but nonetheless the main characteristics have been kept. They include a maximum near 08 LT, a full width at half maximum between 04 LT and 14 LT, and a secondary maximum between 19 LT and 20 LT, which, if confirmed, would indicate a direct generation of accelerated electrons on the duskside. In order to set absolute values for the energy flux, the minimum value was set to zero and the mean value was fixed at the reference level of $0.2 \mathrm{~mW} \mathrm{~m}^{-2}$. The baseline case associated with a constant energy flux equal to the reference level is shown by the dashed line. The incident electrons are assumed to have a Maxwellian distribution in energy with a mean energy $E_{m}$ of $10 \mathrm{keV}$, similar to the hard electron precipitation case illustrated in Figure 3 with dashed lines. The ionospheric and the energy deposition modules of our model, described in section 2, were coupled at a time step of 1 Saturn minute (26.6 Earth seconds), instead of the default 10 Saturn minute time step, in order to capture the LT variation and to infer more precisely the ionospheric response time.

[36] Figures 5b and 5c show the Pedersen and Hall conductances, respectively, for the LT-dependent case (solid line) and the baseline case (dashed line). The conductance ratios between $12 \mathrm{LT}$ and $00 \mathrm{LT}$ are 1.03 and 1.01 for Pedersen and Hall, respectively, for the baseline case. The conductances are nearly proportional to the square root of the energy flux of the incident auroral electrons, $Q_{0}$ (dotted line). This is expected as the conductances are roughly 


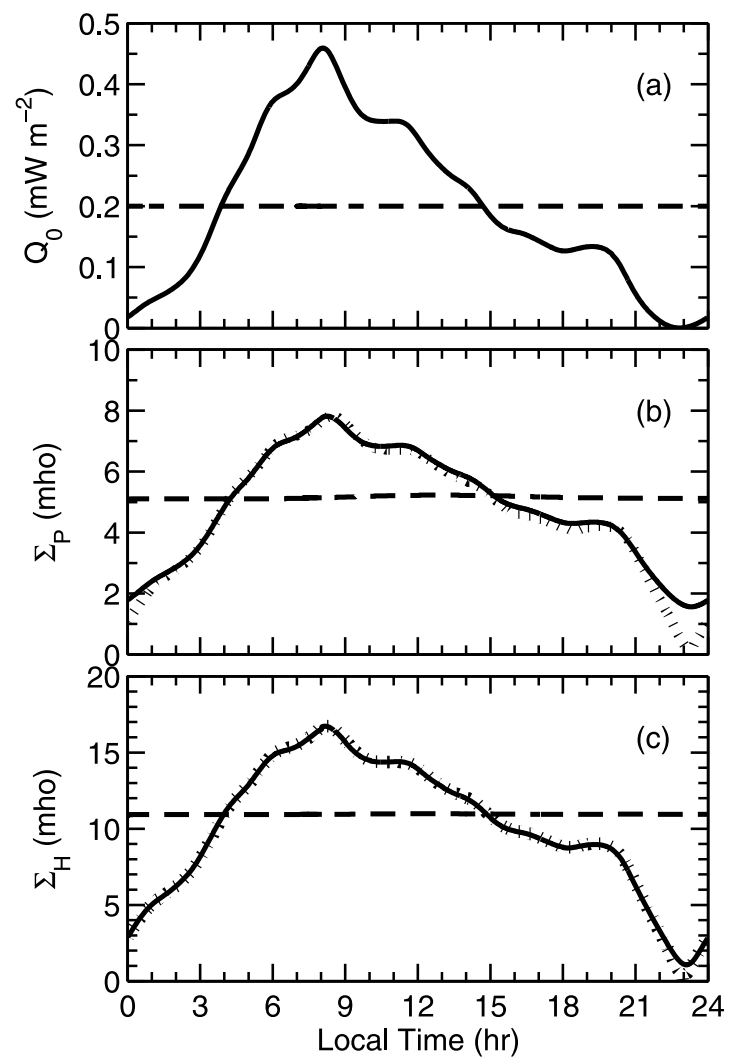

Figure 5. (a) Local time dependence of the energy flux of the incident auroral electrons, $Q_{0}$, assumed to have a Maxwellian distribution in energy and a mean energy, $E_{m}$, of $10 \mathrm{keV}$ (solid line). The LT relative variation is based on the variation of the UV brightness inferred from the analysis of HST/UV images within the main oval [Lamy et al., 2009]. The absolute values of the energy flux are such that the minimum value is 0 , and the mean value is equal to the reference level of $0.2 \mathrm{~mW} \mathrm{~m}^{-2}$. The baseline case associated with a constant energy flux equal to the reference level is shown as dashed line. (b) Same as Figure 5a but for the Pedersen ionospheric conductances. The energy flux of the incident auroral electrons shifted by 23 Saturn minutes and normalized to the maximum value of the Pedersen conductance is also shown with dotted lines. (c) Same as Figure 5a but for the Hall ionospheric conductances. The energy flux of the incident auroral electrons shifted by 10 Saturn minutes and normalized to the maximum value of the Hall conductance is also shown with dotted lines.

proportional to the main ion density, that is, approximatively proportional to the electron density. Furthermore, the electron density is roughly proportional to the square root of the electron production rate, which is proportional to $Q_{0}$.

[37] There is however a delay in the ionospheric response to the change in energy flux. This delay amounts to 23 Saturn minutes (or 10 Earth min $12 \mathrm{~s}$ ) for the Pedersen conductance and to 10 Saturn minutes (or 4 Earth min $26 \mathrm{~s}$ ) for the Hall conductance. The difference in reaction time between the Pedersen and Hall conductances is explained by the difference in peak altitude of the respective conductivities. Below
$2000 \mathrm{~km}\left(8 \times 10^{-8} \mathrm{mbar}\right)$, the ionosphere is in photochemical regime with chemical loss timescales significantly smaller than the diffusive and horizontal transport timescales. For $10 \mathrm{keV}$ auroral electron precipitation, the Pedersen conductivity peaks near $1000 \mathrm{~km}\left(10^{-5}\right.$ mbar) (see Figure 3e), corresponding to chemical lifetimes of a few 100 s of seconds, while the Hall conductivity peaks near $800 \mathrm{~km}\left(8 \times 10^{-5} \mathrm{mbar}\right)$ (see Figure $\left.3 \mathrm{f}\right)$, corresponding to chemical lifetimes of a few 10 s of seconds.

[38] When the energy flux of the incoming auroral electrons becomes small enough, less than $0.04 \mathrm{~mW} \mathrm{~m}^{-2}$ in the present case, the ion and electron densities near the homopause and below decrease significantly, reduced at $23 \mathrm{LT}$ by a factor of about 10 compared with the values at $21 \mathrm{LT}$ and $02 \mathrm{LT}$. As a result, the chemical lifetimes, which are inversely proportional to the electron density when the dominating loss reaction is electron recombination, increase significantly to a few 1000 Earth s. The Pedersen conductance reaches a minimum near $23 \mathrm{LT}$ of 1.56 mho (solid line in Figure 5b), larger than the predicted zero value based on the auroral energy flux (dotted line in Figure 5b). This value is the result of ionization left over from the recent period of precipitation. It is significantly larger than the solar-driven value which only reaches $0.17 \mathrm{mho}$ at $23 \mathrm{LT}$. The effect on the Hall conductance is not as significant as for the Pedersen case. The Hall conductivity peaks at lower altitude where the chemical lifetimes, less than $1000 \mathrm{~s}$, remain smaller than those associated with the Pedersen peak.

\section{Discussion}

7.1. Dependence of Ionospheric Conductances on the Mean Energy and Energy Flux of the Auroral Electrons

[39] Soft auroral electrons, associated with a mean energy $E_{m}$ of $500 \mathrm{eV}$, deposit their energy at high altitude. As a result, they are efficient at increasing the ionospheric densities, and hence the total electron content (TEC) calculated above $1000 \mathrm{~km}$ [Moore et al., 2010], and heating the ionosphere. However, the electrical, ionospheric conductivities peak in regions below the deposition altitude of these auroral electrons. As a result, in the presence of auroral electrons associated with an energy flux $Q_{0}$ of $0.2 \mathrm{~mW} \mathrm{~m}^{-2}$ or less and with a mean energy $E_{m}$ below $300 \mathrm{eV}$ (Pedersen) or below $500 \mathrm{eV}$ (Hall) (see Figure 4), the electrical conductances at noon are driven by the Sun.

[40] Hard auroral electrons, associated with a mean energy $E_{m}$ of $10 \mathrm{keV}$, deposit their energy below the homopause. As a result, they do not efficiently heat the ionosphere due to strong collisional cooling. In addition, the increase of the ionospheric densities is also not as large as for soft electrons. The fact that soft electrons are more efficient at increasing the electron density than hard electrons is due to the different ion compositions in the region of energy deposition. At high altitudes where soft electrons deposit their energy, the prime ion species is $\mathrm{H}^{+}$which is longer lived than $\mathrm{H}_{3}^{+}$ and hydrocarbon ions dominant at lower altitudes where hard electrons deposit their energy. In terms of conductances, auroral electrons, with an energy flux $Q_{0}$ greater than $0.2 \mathrm{~mW}$ $\mathrm{m}^{-2}$ and a mean energy $E_{m}$ greater than $1 \mathrm{keV}$ (Pedersen) or $2 \mathrm{keV}$ (Hall), greatly affect electrical, ionospheric con- 
ductances (see Figure 4). As a result, in the presence of hard electrons, the electrical conductances are driven by the aurora. In addition, the conductances are found to be proportional to $\sqrt{Q_{0}}$ (see Figure 5). However, the response of the ionosphere to changes in $Q_{0}$ is not instantaneous. We found the following LT dependence of the Pedersen and Hall conductances, assuming precipitating auroral electrons with $E_{m}=10 \mathrm{keV}$ and $Q_{0}>0.04 \mathrm{~mW} \mathrm{~m}^{-2}$ :

$$
\Sigma_{P}(t)=11.5 \sqrt{Q_{0}\left(t-\Delta t_{P}\right)} \quad(\mathrm{mho})
$$

and

$$
\Sigma_{H}(t)=24.7 \sqrt{Q_{0}\left(t-\Delta t_{H}\right)} \quad(\mathrm{mho})
$$

where $\mathrm{Q}_{0}$ is in $\mathrm{mW} \mathrm{m}^{-2}, \Delta t_{P}=23$ Saturn minutes (or 10 Earth min $12 \mathrm{~s}$ ) and $\Delta t_{H}$ is 10 Saturn minutes (or 4 Earth min $26 \mathrm{~s}$ ). The electrical conductivities peak in regions where photochemical equilibrium prevails. As a result, the time delays, $\Delta t_{P}$ and $\Delta t_{H}$, are sensitive to the chemical timescales in the region where the conductivity profiles peak, which varies with $E_{m}$, especially in the Pedersen case (see Figure 3e). Timescales are also affected by the presence and magnitude of the energy sources. For other $\mathrm{E}_{m}$ values, the proportionality coefficients can be derived from Figure 4 valid for $\mathrm{Q}_{0}=0.2 \mathrm{~mW} \mathrm{~m}^{-2}$.

[41] Our calculations of the Hall conductance and associated time delay $\Delta t_{H}$ below the homopause remain limited because of the simplified chemical model that we adopted below the homopause. Although the calculated total electron density is close to that obtained with a comprehensive ionospheric model, the main ion species we assume is $\mathrm{CH}_{5}^{+}$, instead of $\mathrm{C}_{3} \mathrm{H}_{5}^{+}$[Moore et al., 2008]. This simplified chemistry affects to a much lesser extent the calculations of the Pedersen conductance with an error of less than 0.06 mho $(0.6 \mathrm{mho})$ for electron densities less than $10^{3} \mathrm{~cm}^{-3}\left(10^{4} \mathrm{~cm}^{-3}\right)$ [Moore et al., 2010], as the associated conductivity peaks near or above the homopause (see Figure 3e). Furthermore, Moses and Bass [2000], who included the full hydrocarbon photochemistry in their ionospheric model, pointed out that the contribution by the hydrocarbons at noon is less than $5 \%$ of the total conductance under solar-driven conditions.

[42] The incident auroral electrons have been assumed to have a Maxwellian distribution in energy. For a mean energy $E_{m}$ of $10 \mathrm{keV}$ and an energy flux $Q_{0}$ of $0.2 \mathrm{~mW} \mathrm{~m}^{-2}$, the conductances are changed by less than $1 \%$ between the case of a Maxwellian distribution and the one of a Kappa distribution with a Kappa coefficient of 3 for the incident electrons. However, the difference increases with lower $E_{m}$, as anticipated from Figure A1. For $E_{m}=2 \mathrm{keV}$, the Kappato-Maxwellian conductance ratio reaches 0.8 and 1.7 for Pedersen and Hall conductivities, respectively. These differences are explained by the presence of the high-energy tail in the case of the Kappa distribution, which induces a smaller Pedersen conductivity peak and a larger Hall conductivity peak for a Kappa distribution compared with a Maxwellian distribution.

\subsection{Comparison With Other Modeling Studies}

[43] Cowley et al. [2008] derived a value of 4 mho for the effective Pedersen conductance $\Sigma_{P}^{*}$, defined as $\Sigma_{P}^{*}=$ $(1-k) \Sigma_{P}$ [Cowley et al., 2004b]. The parameter $k$ between
0 and 1 is introduced in order to take account of the possible slippage of the neutral atmosphere from rigid corotation due to ion-neutral frictional drag [Bunce et al., 2003]. It is defined as [Cowley et al., 2004b]

$$
k=\frac{\Omega-\omega_{n}}{\Omega-\omega_{i}}
$$

where $\Omega$ is the planetary angular velocity, $\omega_{n}$ is the angular velocity of the neutral atmosphere, and $\omega_{i}$ is the angular velocity of the plasma. We do not need to introduce an effective conductance in our calculations as the ion-neutral drag and associated neutral dynamics are included in the model and taken into account when calculating the Joule heating. The value of $k$ has previously not been calculated for Saturn. Based on simulations with the Jovian Ionosphere Model (JIM), a value of $k \approx 0.5$ calculated for Jupiter by G. Millward was adopted also for Saturn [Cowley et al., $2004 b]$. The STIM model enables us to calculate $k$, and in our simulations we find $k \approx 0.5$ also for Saturn. We find no noticeable variation of $k$ from this value for the different electric field strengths we assumed in this study.

[44] The value of 4 mho for $\Sigma_{P}^{*}$ proposed for summer solstice conditions with an inclination of $14^{\circ}$ [Cowley et al., 2008] thus yields a value for $\Sigma_{P}$ of 8 mho. For $10 \mathrm{keV}$ electrons, this corresponds to a value of $0.48 \mathrm{~mW} \mathrm{~m}^{-2}$ for equinox (from relation (5)) and of $0.42 \mathrm{~mW} \mathrm{~m}^{-2}$ for summer solstice with an inclination of $23.7^{\circ}$ (from section 7.4 and relation (5)). These values of energy flux are consistent with values derived from the analysis of UV emissions for quiet conditions (see section 3).

[45] It should be noted that the values obtained for ionospheric conductances at Saturn in the auroral regions are significantly larger than the values obtained at Earth and Jupiter. At Earth, Pedersen and Hall conductances are found to be 4-6 mho and 7-11 mho, respectively, for a characteristics energy of $5 \mathrm{keV}$ (mean energy of $10 \mathrm{keV}$ for Maxwellian distribution) and an energy flux of $1 \mathrm{~mW} \mathrm{~m}^{-2}$ [Fuller-Rowell and Evans, 1987]. These values are about half those found at Saturn (based on relations (5) and (6)). The main difference between Earth and Saturn is the altitude range over which conductivities are peaking. The altitude region, over which values of conductivities are at least a tenth of the peak value, is about $70 \mathrm{~km}$ (Pedersen) and $50 \mathrm{~km}$ (Hall) at Earth and $500 \mathrm{~km}$ (Pedersen) and 300 $400 \mathrm{~km}$ (Hall) at Saturn. A reduction by a factor of 10 of the region where conductivities peak yields a reduction by the same factor of the conductances. Another difference between Earth and Saturn is the composition of the atmosphere. Conductivities are sensitive to neutral and ion composition [e.g., Hinson et al., 1998; Moore et al., 2010]. At Jupiter, for $10 \mathrm{keV}$ electrons with an energy flux of $1 \mathrm{~mW} \mathrm{~m}^{-2}$ the Pedersen and Hall conductivities peak at values smaller than $10^{-6} \mathrm{mho} \mathrm{m}^{-1}$ and $10^{-8} \mathrm{mho} \mathrm{m}^{-1}$, respectively [Millward et al., 2002] (to be compared with the Saturn case illustrated with dashed lines in Figures $3 \mathrm{e}$ and $3 \mathrm{f}$ valid for an energy flux of $0.2 \mathrm{~mW} \mathrm{~m}^{-2}$ ). This yields values for the Pedersen and Hall conductances of $0.1-0.2$ mho and $2 \times 10^{-3}$ mho, respectively, significantly smaller than those found at Saturn (11.5 mho and 24.7 mho for Pedersen and Hall conductances, respectively, for an energy flux of $1 \mathrm{~mW} \mathrm{~m}^{-2}$ and a mean energy of $10 \mathrm{keV}$ (based on relations (5) and (6)). Atmospheric 
Table 1. Comparison of Calculated and Observed Effective $\mathrm{H}_{3}^{+}$Column Temperature $T_{F}$ and Density $N_{F}{ }^{\mathrm{a}}$

\begin{tabular}{|c|c|c|c|c|c|c|c|}
\hline & \multicolumn{5}{|c|}{ STIM Modeling (Sun + Aurora) } & \multicolumn{2}{|c|}{ UKIRT Observations $^{\mathrm{b}}$} \\
\hline & Sun Only & $\mathrm{E}_{m}=150 \mathrm{eV}$ & $\mathrm{E}_{m}=500 \mathrm{eV}$ & $\mathrm{E}_{m}=2 \mathrm{keV}$ & $\mathrm{E}_{m}=10 \mathrm{keV}$ & 1999 & 2004 \\
\hline$T_{F}(\mathrm{~K})$ & 500 & 506 & 502 & $\begin{array}{l}485 \\
450^{\mathrm{c}}\end{array}$ & 484 & $380 \pm 70$ & $420 \pm 70$ \\
\hline$N_{F}\left(10^{12} \mathrm{~cm}^{-2}\right)$ & 0.23 & 0.72 & 0.69 & $\begin{array}{l}0.46 \\
0.31^{\mathrm{c}}\end{array}$ & 0.34 & $\begin{array}{c}1.9 \pm 0.2^{\mathrm{d}}\left(\mathrm{T}_{F}=380 \mathrm{~K}\right) \\
0.5\left(\mathrm{~T}_{F}=450 \mathrm{~K}\right)\end{array}$ & $\begin{array}{c}7.3 \pm 0.7^{\mathrm{d}}\left(\mathrm{T}_{F}=420 \mathrm{~K}\right) \\
2.5\left(\mathrm{~T}_{F}=490 \mathrm{~K}\right)\end{array}$ \\
\hline$N_{M}\left(10^{12} \mathrm{~cm}^{-2}\right)$ & 0.36 & 1.71 & 1.10 & $\begin{array}{l}0.68 \\
0.65^{\mathrm{d}}\end{array}$ & 0.53 & - & - \\
\hline
\end{tabular}

${ }^{\mathrm{a}}$ The calculated values (columns 2-6) are derived from the ratio between the $\nu_{2} \mathrm{R}\left(1,0^{-}\right)$line $(3.669 \mu \mathrm{m})$ and the $\nu_{2} \mathrm{R}\left(3,3^{-}\right)$line $(3.534 \mu \mathrm{m})$ and are valid under solar illumination for $78^{\circ} \mathrm{S}$ at noon, assuming equinox and solar minimum conditions. The incident electrons have a Maxwellian distribution with a given mean energy $\mathrm{E}_{m}$ and an energy flux $\mathrm{Q}_{0}$ of $0.2 \mathrm{~mW} \mathrm{~m}^{-2}$. The $\mathrm{H}_{3}^{+}$column densities $N_{M}$ are obtained by integrating the $\mathrm{STIM}$-calculated $\mathrm{H}_{3}^{+}$number density over the whole altitude range. The effective $\mathrm{H}_{3}^{+}$temperatures and column densities derived by fitting $\nu_{2} \mathrm{R}$ branch emission lines of $\mathrm{H}_{3}^{+}$observed with UKIRT are given in the last two columns [Melin et al., 2007].

${ }^{\mathrm{b}}$ Assuming quasi-local thermodynamic equilibrium [Miller et al., 1990].

${ }^{c}$ Using thermospheric density profiles associated with an exospheric temperature $\mathrm{T}_{\text {exo }}$ of $485 \mathrm{~K}$.

${ }^{\mathrm{d}}$ Given with a $3 \sigma$ error.

composition and integration region for the conductivities are similar at Jupiter and Saturn. The major difference between these two planets is the magnetic field strength. Jupiter's magnetic field, the strongest planetary field encountered in the Solar System, is 20 (14) times as strong as Saturn's (Earth's). The conductances are dependent on the magnetic field strength through the angular gyrofrequency. If the magnetic field of Saturn is multiplied by a factor 20, the conductances are found to decrease by a factor 150 to 200 . While a more systematic study should be carried out, this preliminary comparison between Jupiter, Saturn, and Earth highlights the major differences between the three planets. This is anticipated to have implications on the magnetosphere-ionosphere coupling, in particular on the energy of electrons accelerated along magnetic field lines.

\subsection{Comparison With Observations}

[46] A full comparison of STIM modeling outputs and Cassini/RSS measurements has been presented by Moore et al. [2010]. It covers the full latitude range and discusses the sensitivity of input parameters, such as the reaction rate $\mathrm{k}_{1}^{*}$ between $\mathrm{H}_{2}(\nu \leq 4)$ and $\mathrm{H}^{+}$, composition, season, and solar activity. Several radio occultation observations, which provide electron density profiles, occurred within the variable auroral boundaries of the main oval between $70^{\circ}$ and $85^{\circ}$ [Badman et al., 2006]: one by Voyager 1/Radio Science Subsystem (RSS) [Lindal et al., 1985] and three by Cassini/ RSS [Kliore et al., 2009]. The Voyager 1 profile $\left(73^{\circ} \mathrm{S}\right)$ and the Cassini $056 \mathrm{x}$ profile $\left(71.8^{\circ} \mathrm{S}\right)$ exhibit two peaks between $1200 \mathrm{~km}$ and $2000 \mathrm{~km}$ with magnitude between $10^{4}$ and $2 \times$ $10^{4} \mathrm{~cm}^{-3}$. These peaks are close to, though weaker than, those predicted by our model for soft electron precipitation (see Figure 3c). The weakness of the observed values may be explained by the rather equatorward latitude of the observations and by the late local time of the observations, which is mostly associated with weak auroral brightnesses [e.g., Cowley et al., 2004a; Grodent et al., 2005; Lamy et al., 2009].

[47] The electron density profiles derived from the two other Cassini occultations, $044 \mathrm{n}\left(75.4^{\circ} \mathrm{N}\right)$ and $058 \mathrm{x}\left(74.1^{\circ} \mathrm{S}\right)$, only extend down to $1500 \mathrm{~km}$ [Moore et al., 2010]. Over this range, they exhibit peaks between $1700-2000 \mathrm{~km}$ of magnitude between 3-4 $410^{4} \mathrm{~cm}^{-3}$ [Kliore et al., 2009]. Such high-altitude peaks attest to the presence of soft auroral electrons. HST observations of Saturn southern aurora occurred 12 hours prior to and 14 hours after the $058 \mathrm{x}$ occultation and reveal that the auroral dusk region sampled by $058 \mathrm{x}$ was particularly quiet [Moore et al., 2010]. No auroral image associated with $044 \mathrm{n}$ is available. As for upcoming campaigns by Cassini, unfortunately no radio occultation at a latitude above $70^{\circ}$ is planned before the end of the Cassini mission (A. Kliore, personal communication, 2010).

[48] Another type of observations relevant for comparison with the modeling is the measurement of $\mathrm{H}_{3}^{+}$vibrational emissions in the infrared. Melin et al. [2007] analyzed observations of the high-latitude regions of Saturn acquired with the facility spectrometer, CGS4, on the United Kingdom Infrared Telescope (UKIRT), Mauna Kea, Hawaii. Observed emission lines from the $\mathrm{R}$ branch of the $\mathrm{H}_{3}^{+}$fundamental $\nu_{2}$ manifold were used to derive effective $\mathrm{H}_{3}^{+}$column temperatures $\mathrm{T}_{F}$ and column densities $\mathrm{N}_{F}$. In order to compare the STIM simulations with these observations, we have used the $\mathrm{H}_{3}^{+}$column emission model developed by Miller et al. [2010]. Such a model computes the effective $\mathrm{H}_{3}^{+}$column temperature and density from the ratio of two given emission lines. The inputs include the molecular hydrogen density, the thermospheric temperature, and the $\mathrm{H}_{3}^{+}$density, all outputs of the STIM model. The modeled values for $\mathrm{T}_{F}$ and $\mathrm{N}_{F}$ derived from the ratio between $\nu_{2} \mathrm{R}\left(1,0^{-}\right)$line at $3.669 \mu \mathrm{m}$ and the $\mathrm{R}\left(3,3^{-}\right)$line at $3.534 \mu \mathrm{m}$ are presented for different mean energies of incident electrons in Table 1. The last line of the table provides the $\mathrm{H}_{3}^{+}$column densities $\mathrm{N}_{M}$ obtained by integrating the STIM $\mathrm{H}_{3}^{+}$profile over the full altitude range. The STIM effective column densities $\mathrm{N}_{F}$ are always lower than the STIM $\mathrm{N}_{M}$, as a result of the quasilocal thermodynamic equilibrium populations of the vibrationally excited levels. $\mathrm{N}_{F}$ corresponds to an apparent column density, while $\mathrm{N}_{M}$ provides a better estimate of the "true" column density. Result of the analysis of the 1999 and 2004 observation campaigns are given in the last two columns of Table 1. For each observational campaign, two sets of column densities are given, one associated with $\mathrm{T}_{F}$ equal to $380 \mathrm{~K}$ and the other, with $\mathrm{T}_{F}$ equal to $450 \mathrm{~K}$. This highlights the sensitivity of the derived column density with the uncertainty in $\mathrm{T}_{F}$. Values are significantly larger for the 2004 campaign which is associated with very strong auroral activity. There- 
fore, as the STIM simulations were performed for quiet, auroral conditions, they should be compared with the 1999 campaign.

[49] The $\mathrm{H}_{3}^{+}$column temperatures $\mathrm{T}_{F}$ and column densities $\mathrm{N}_{F}$ modeled and those observed in 1999 are of the same order of magnitude. The STIM $\mathrm{T}_{F}$ values are slightly larger than the observed ones. The exospheric temperature used for the calculations is $510 \mathrm{~K}$ (and an energy flux of $0.2 \mathrm{~mW}$ $\mathrm{m}^{-2}$ ). For a thermospheric temperature reduced to $485 \mathrm{~K}$, the inferred $\mathrm{T}_{F}$ for $2 \mathrm{keV}$ electrons is found to be $450 \mathrm{~K}$, upper limit of the values inferred from observations. The STIM column densities $\mathrm{N}_{F}$ agree well with the values derived from observations when the larger value of $\mathrm{T}_{F}$ is used for inferring $\mathrm{N}_{F}$. Using an exospheric temperature of $485 \mathrm{~K}$, the column density $\mathrm{N}_{F}$ derived for $2 \mathrm{keV}$ electrons is reduced to $0.31 \times 10^{12} \mathrm{~cm}^{-2}$. This seems to indicate that the energy flux of the incoming electrons was more intense than the $0.2 \mathrm{~mW} \mathrm{~m}^{-2}$ value considered here. If the energy flux is increased from 0.2 to $2 \mathrm{~mW} \mathrm{~m}^{-2}\left(10 \mathrm{~mW} \mathrm{~m}^{-2}\right)$, the $\mathrm{H}_{3}^{+}$column temperature decreases by $3 \%(4.7 \%)$ and the $\mathrm{H}_{3}^{+}$column density $\mathrm{N}_{F}$ increases by a factor 1.4 (1.9) to $0.43 \times 10^{12} \mathrm{~cm}^{-2}\left(0.59 \times 10^{12} \mathrm{~cm}^{-2}\right)$. The $\mathrm{N}_{M}$ column densities increase from $0.53 \times 10^{12} \mathrm{~cm}^{-2}$ for $\mathrm{Q}_{0}=0.2 \mathrm{~mW} \mathrm{~m}^{-2}$ to $0.96 \times 10^{12} \mathrm{~cm}^{-2}\left(1.63 \times 10^{12} \mathrm{~cm}^{-2}\right)$ for $\mathrm{Q}_{0}=2 \mathrm{~mW} \mathrm{~m}$ $\left(10 \mathrm{~mW} \mathrm{~m}^{-2}\right)$. The increase in $\mathrm{N}_{F}$ is modest compare with the increase in $\mathrm{Q}_{0}$ because increasing $\mathrm{Q}_{0}$ yields an increase in electron density and in general $\mathrm{H}^{+} / \mathrm{H}_{3}^{+}$ratio is proportional to the electron density [e.g., Moore et al., 2004]. Based on these simulations, the $\mathrm{H}_{3}^{+}$column temperatures and densities derived from the 1999 campaign are consistent with an exospheric temperature at $485 \mathrm{~K}$ or colder and precipitating electrons with a mean energy smaller than a few $\mathrm{keV}$.

[50] In the future, we envisage to carry out similar comparisons using the Cassini/VIMS $\mathrm{H}_{3}^{+}$auroral data set [Stallard et al., 2008c] and to perform dedicated runs for the specific events analyzed.

\subsection{Sensitivity of the Ionospheric Conductances With Solar and Seasonal Conditions}

[51] Under solar illumination, in the absence of hard electron precipitation, Pedersen and Hall ionospheric conductances vary with solar activity and with season. At noon in the absence of particle precipitation, the solar maximum to solar minimum conductance ratio is found to be 1.6 for Pedersen and 1.9 for Hall. The larger increase with solar activity of Hall conductance compared with Pedersen conductance is explained by the larger increase in solar soft $\mathrm{X}$-rays, depositing their energy at low altitudes where Hall conductivity peaks, compared with solar EUV photons, depositing their energy mostly at higher altitudes. In addition, at a latitude of $78^{\circ}$ at noon, the SZA increases from $78^{\circ}$ at equinox to $51^{\circ}$ at summer solstice. At solar minimum conditions and in the absence of particle precipitation, the summer solstice to equinox conductance ratio at $78^{\circ} \mathrm{S}$ is found to be 2.6. Despite this significant variation with season, conductances remain proportional to the energy flux $Q_{0}$ in the presence of hard electron precipitation. The conductance ratio at summer solstice between noon and midnight under $10 \mathrm{keV}$ electron precipitation with $Q_{0}=0.2 \mathrm{~mW} \mathrm{~m}^{-2}$ is less than 1.05. The solar contribution however influences the absolute magnitude of the conductances with the proportionality factor in relations (5) and (6) increased to 12.4 (Pedersen) and 24.4 (Hall), respectively, at summer solstice.

[52] An intrinsic asymmetry is present between the northern hemisphere $(\mathrm{NH})$ and the southern hemisphere $(\mathrm{SH})$ due to the magnetic field strength and dip angle whose values at a given latitude depends on the hemisphere considered. We find that the conductances are reduced in the $\mathrm{NH}$ compared with the $\mathrm{SH}$. At equinox, $\mathrm{SH}$ to $\mathrm{NH}$ conductance ratios of 1.2 and 1.3 are found for Pedersen and Hall conductivities, respectively, assuming the same, default auroral conditions $\left(E_{m}=10 \mathrm{keV}, Q_{0}=0.2 \mathrm{~mW} \mathrm{~m}^{-2}\right.$, electric field as defined in section 4). However, the ionospheric state affects the magnetospheric forcing. Different values in ionospheric conductances between the two hemispheres due to the magnetic field asymmetry may yield different values for the precipitating electron characteristics $\left(E_{m}\right.$ and $\left.Q_{0}\right)$ and for the electric field. This asymmetry may be reinforced at southern, summer solstice, especially if the precipitating particles are soft.

\subsection{Sensitivity of the Ionospheric Conductances With Chemical and Thermospheric Conditions}

[53] There are uncertainties in the effective reaction rate, $\mathrm{k}_{1}^{*}$, associated with charge exchange between $\mathrm{H}^{+}$and vibrationally excited $\mathrm{H}_{2}$ (see relation (2)). While the actual reaction rate $\mathrm{k}_{1}$ is now well established [e.g., Huestis, 2008], the fraction of vibrationally excited $\mathrm{H}_{2}$ remains uncertain. We have taken the latter to be twice that proposed by Moses and Bass [2000]. Moore et al. [2010] showed that at low and middle latitudes, the best fit of the modeled total electron content (TEC) with those derived from RSS radio occultations is reached for an effective reaction rate equal to $0.125 \mathrm{k}_{1}^{*}$ (or a fraction of $\mathrm{H}_{2}(\nu \geq 4)$ equal to 0.25 that of Moses and Bass [2000]). In these regions, the dominant source for $\mathrm{H}_{2}$ $(\nu \geq 4)$ is primarily fluorescence [Majeed et al., 1991]. The reason for choosing $k_{1}^{*}$ (and not the reduced value) in the auroral regions is justified by the larger source of $\mathrm{H}_{2}(\nu \geq 4)$ occurring in these regions as a result of energetic electron precipitation through direct vibrational excitation by electron impact [Allan, 1985], cascading associated with Lyman and Werner band excitation by electron impact, and dissociative recombination of $\mathrm{H}_{3}^{+}$, as shown at Jupiter [Cravens, 1987]. Though loss of $\mathrm{H}_{2}(\nu \geq 4)$ is increased through vibration translation collisions with $\mathrm{H}$ which builds up in the auroral region, the net effect is an increase in $\mathrm{H}_{2}(\nu \geq 4)$ density [Cravens, 1987]. It is nevertheless useful to assess the sensitivity of the ionospheric conductances to this parameter.

[54] Under solar illumination alone, Pedersen and Hall conductances at $12 \mathrm{LT}$ change by $2 \%$ and less than $0.1 \%$, respectively, when $\mathrm{k}_{1}^{*}$ is multiplied by 0.25 . At high latitude, in the absence of aurora, the electron density is modest (see Figure 3c). As the $\mathrm{H}^{+}$to $\mathrm{H}_{3}^{+}$density ratio is proportional to the total electron density [e.g., Moore et al., 2004], the electron density there does not strongly depend on $\mathrm{k}_{1}^{*}$. When hard electron precipitation occurs, the change in electron density with $\mathrm{k}_{1}^{*}$ also remains modest as the energy deposition occurs at low altitudes where $\mathrm{H}^{+}$is not the major ion. For incident electrons with a mean energy of $10 \mathrm{keV}$, the Pedersen and Hall conductances change by a factor of $5 \%$ and $0.1 \%$, respectively, when $\mathrm{k}_{1}^{*}$ is multiplied by 0.25 . The 


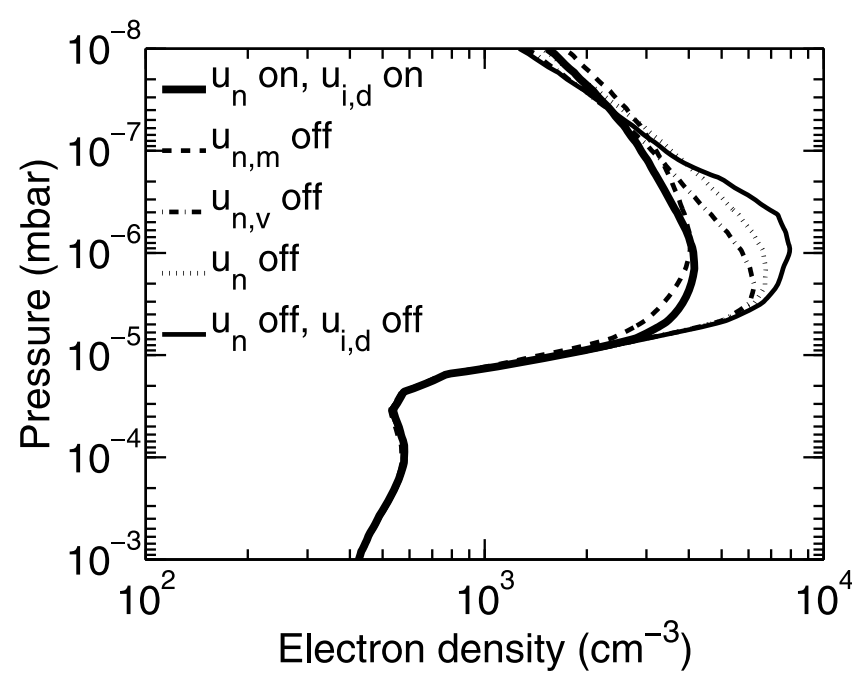

Figure 6. Electron density from STIM calculations at $78^{\circ} \mathrm{S}$ latitude, at noon (SZA of $78^{\circ}$ ), at equinox, during solar minimum conditions, under solar illumination in the absence of electron precipitation, with both thermospheric wind $\mathrm{u}_{n}$ and plasma diffusion drift $\mathrm{u}_{i, d}$ considered for deriving the ion drift (thick, solid line), with the meridional, thermospheric wind $\mathrm{u}_{n, m}$ turned off (dashed line), with the vertical, thermospheric wind $\mathrm{u}_{n, v}$ turned off (dash-dotted line), with the total thermospheric wind turned off (dotted line), and with the total ion drift $\left(\mathrm{u}_{n}\right.$ and $\left.\mathrm{u}_{i, d}\right)$ turned off (thin, solid line).

effect increases with decreasing mean energy. For incident electrons with a mean energy of $2 \mathrm{keV}$, the Pedersen and Hall conductances change by a factor of $10 \%$ and $0.6 \%$, respectively, when $\mathrm{k}_{1}^{*}$ is multiplied by 0.25 .

[55] We find that the ionospheric conductances are not very sensitive to the atomic hydrogen or helium mixing ratio. At noon under pure solar illumination, Pedersen and Hall conductances vary by less than $2 \%$ for an increase of a factor 10 in the atomic hydrogen and by less than $9 \%$ and $3 \%$, respectively, for an increase of a factor of 5 in the helium density.

[56] As on Earth, we find thermospheric dynamics at Saturn to influence the electron densities and, to a certain extent, the conductances. The ion drift, which is included in the continuity equations solved along the local vertical, has three components: the meridional $\left(u_{n, m}\right)$ and the vertical $\left(u_{n, v}\right)$, thermospheric winds and the plasma diffusion [Rishbeth and Garriott, 1969; Moore et al., 2004]. The thermospheric winds are extracted from the 3-D STIM model at each local time considered. In the presence of an equatorward electric convection field, as assumed in our simulations, ions are accelerated westward in the $\mathbf{E} \times \mathbf{B}$ direction, which via ion-neutral collisions introduces an additional westward acceleration to the thermospheric neutrals as well. Joule heating, driven by Pedersen currents, locally heats thermospheric gases and drives meridional acceleration. Divergence of meridional and zonal winds yields vertical wind velocities of typically several $\mathrm{m} \mathrm{s}^{-1}$, but reaching values up to tens of $\mathrm{m} \mathrm{s}^{-1}$ within the auroral oval. When projected along the magnetic field line, the contribution of the meridional wind $\mathrm{u}_{n, m}$ is minor due to the large dip angle at high latitudes, here $78^{\circ}$. The main contributors to the ion drift are found to be the thermospheric vertical winds and plasma diffusion, which affect the vertical distribution of plasma, as illustrated in Figure 6. However, as the conductivities peak at high pressures (see Figure 3), the effect of dynamics on the conductances is moderate, at least for the solar case in the absence of electron precipitation. For the pure solar case the Pedersen and Hall conductances at $12 \mathrm{LT}$ are changed by less than $15 \%$ and $1 \%$, respectively, if the ion drift (or one of its component) is ignored (compared with the case for which the ion drift is considered in the simulation).

[57] There currently are no constraints on some physical quantities affecting the calculation of the ionospheric conductances, such as the vertical, thermospheric winds, or large uncertainties on them, such as the effective reaction rate $k_{1}^{*}$. The baseline value of the conductances, especially Pedersen conductance, may therefore vary. Nevertheless the sensitivity study proposed in section 7 should give insight when these parameters are changed. The general trends highlighted in this paper remain valid.

\section{Appendix A: Validation of Auroral Electron Deposition at a Giant Planet}

[58] In order to validate our auroral electron transport calculations in a giant planet atmosphere, we first compare our modeling output with other findings presented in the literature. We assume a pure $\mathrm{H}_{2}$ atmosphere. The $\mathrm{H}_{2}$ column density we derived between the top of the atmosphere and the altitude of the energy deposition peak is plotted as a function of the mean energy of the incident electrons in Figure A1. Under the pure $\mathrm{H}_{2}$ assumption, Figure $\mathrm{A} 1$ is valid for any giant planet. The larger the incident electron energy, the lower the altitude of deposition, and therefore the higher the associated $\mathrm{H}_{2}$ column density. The incident electrons are assumed to have a Gaussian distribution in energy. For comparison, the results obtained by Gustin et al.

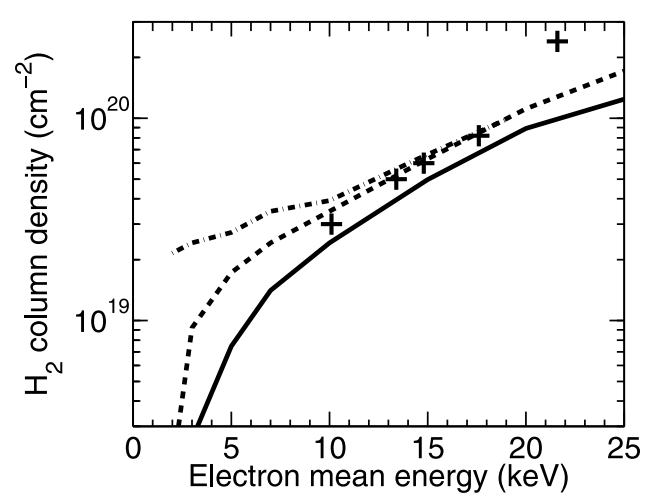

Figure A1. Column density of $\mathrm{H}_{2}$ between the altitude of maximum energy deposition and the top of the atmosphere as a function of the mean energy of the incident auroral electrons, assuming a Gaussian distribution (solid line), a Maxwellian distribution (dashed line), and a Kappa distribution with a Kappa parameter of 3 (dash-dotted line). The atmosphere is assumed to be only composed of $\mathrm{H}_{2}$. The results from Gustin et al. [2009, Table 1] are shown with crosses and are associated with a monoenergetic distribution in energy. 


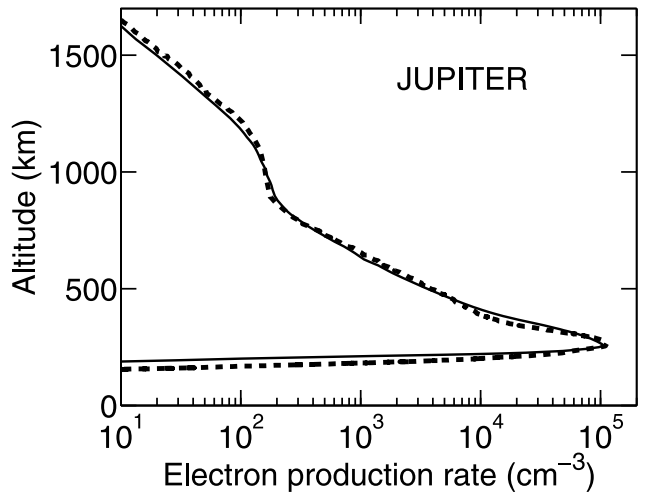

Figure A2. Profile in altitude of the electron production rate (solid line) assuming the same triple Maxwellian distribution for the incident auroral electrons as Grodent et al. [2001] and similar thermospheric conditions at Jupiter. For reference, the profile of the electron production rate computed by Grodent et al. [2001] is shown as dashed line.

[2009], who assumed a monoenergetic distribution in energy in a pure $\mathrm{H}_{2}$ atmosphere, are shown with crosses. There is a good agreement, within $20 \%$, between the $\mathrm{H}_{2}$ column density values we computed (solid line) and those obtained by Gustin et al. [2009]. The only exception is for the case above $20 \mathrm{keV}$ for which the discrepancy reaches $60 \%$. It is not clear what the reason is, as the electron impact cross sections for $\mathrm{H}_{2}$ do not show any dramatic change in this energy range and therefore, there is no apparent reason for a sharp change as seen in the work by Gustin et al. [2009] results.

[59] Furthermore, we confirm the finding of Gustin et al. [2009] that over the $10-20 \mathrm{keV}$ range, the difference in terms of $\mathrm{H}_{2}$ column density at the altitude of maximum energy deposition between the Gaussian (or monoenergetic) distribution (solid line) and the Maxwellian distribution (dashed line) is small, within 25 to $30 \%$. This remains valid also when a Kappa distribution (dash-dotted line) is considered which provides $\mathrm{H}_{2}$ column density within $10 \%$ of the values for a Maxwellian distribution. However, at lower energies, the $\mathrm{H}_{2}$ column density becomes more sensitive to the energy distribution of the incident electrons. The $\mathrm{H}_{2}$ column density value obtained for a Maxwellian distribution is a factor of 3.6 and 2.3 greater than the one derived for a Gaussian distribution for a mean energy of $3 \mathrm{keV}$ and $5 \mathrm{keV}$, respectively. As more energetic electrons need to encounter a larger column density before depositing their energy, the $\mathrm{H}_{2}$ column density value at maximum deposition is larger for a broad Maxwellian distribution compared with a narrow Gaussian distribution. The difference in $\mathrm{H}_{2}$ column density at low energies is even larger when a Kappa distribution is assumed for the incident electrons. The high-energy power law tail of the Kappa distribution associated here with a Kappa parameter of 3 induces a value for the $\mathrm{H}_{2}$ column density at $3 \mathrm{keV}$ a factor of 2.6 and 9.1 times larger than the values obtained with the Maxwellian distribution and a Gaussian distribution, respectively. The choice of energy distribution for the incident electron has therefore a signif- icant impact on the altitude of deposition for low-energy electrons $(<5 \mathrm{keV})$ and thus on the energy of the incident electrons derived from auroral emission analysis.

[60] Figure A2 shows a comparison between the electron production rate we calculated (solid line) and that derived by Grodent et al. [2001] (dashed line). The incident electrons are assumed to have a triple Maxwellian distribution in energy as defined by Grodent et al. [2001]. The thermospheric model we used as input is the same as the one given by Grodent et al. [2001]. The altitude profile of the electron production rate we compute agrees very well with that produced by Grodent et al. [2001], which provides a validation of our auroral electron energy deposition model.

[61] Acknowledgments. This work was supported by a grant from the NASA Cassini Data Analysis Program (CDAP) to Boston University. M.G. was partially supported by the Science and Technology Facilities Council (STFC) rolling grant to Imperial College London. I.M.W. was funded by a Royal Society University Research Fellowship. We would like to thank Arv Kliore, Tom Stallard, and Nick Achilleos very much for constructive discussions. We would like to acknowledge ISSI for the great opportunity it offered us as well as the members of the ISSI International Team on Saturn Aeronomy (166) for very valuable discussions on this topic.

[62] Robert Lysak thanks the reviewers for their assistance in evaluating this paper.

\section{References}

Allan, M. (1985), Experimental observation of structures in the energy dependence of vibrational excitation in $\mathrm{H}_{2}$ by electron impact in the ${ }^{2} \Sigma_{u}^{+}$resonance region, J. Phys. B At. Mol. Phys., 18, L451-L455.

Anderson, J. D., and G. Schubert (2007), Saturn's gravitational field, internal rotation, and interior structure, Science, 317, 1384-1387, doi:10.1126/ science. 1144835

Badman, S. V., S. W. H. Cowley, J.-C. Gérard, and D. Grodent (2006), A statistical analysis of the location and width of Saturn's southern auroras, Ann. Geophys., 24, 3533-3545.

Banks, P. M., and G. Kockarts (1973), Aeronomy, Elsevier, New York.

Bunce, E. J., S. W. H. Cowley, and J. A. Wild (2003), Azimuthal magnetic fields in Saturn's magnetosphere: Effects associated with plasma subcorotation and the magnetopause-tail current system, Ann. Geophys., 21, 1709-1722.

Clarke, J.-T., et al. (2005), Morphological differences between Saturn's ultraviolet aurorae and those of Earth and Jupiter, Nature, 433, 717-719, doi:10.1038/nature03331.

Connerney, J. E. P., and J. H. Waite Jr. (1984), New model of Saturn's ionosphere with an influx of water from the rings, Nature, 312, 136-138.

Cowley, S. W. H., and E. J. Bunce (2001), Origin of the main auroral oval in Jupiter's coupled magnetosphere-ionosphere system, Planet. Space Sci., 49, 1067-1088, doi:10.1016/S0032-0633(00)00167-7.

Cowley, S. W. H., E. J. Bunce, and R. Prangé (2004a), Saturn's polar ionospheric flows and their relation to the main auroral oval, Ann. Geophys., 22, 1379-1394.

Cowley, S. W. H., E. J. Bunce, and J. M. O'Rourke (2004b), A simple quantitative model of plasma flows and currents in Saturn's polar ionosphere, J. Geophys. Res., 109, A05212, doi:10.1029/2003JA010375.

Cowley, S. W. H., et al. (2008), Auroral current systems in Saturn's magnetosphere: Comparison of theoretical models with Cassini and HST observations, Ann. Geophys., 26, 2613-2630.

Cravens, T. E. (1987), Vibrationally excited molecular hydrogen in the upper atmosphere of Jupiter, J. Geophys. Res., 92, 11,083-11,100.

Davis, L., Jr., and E. J. Smith (1990), A model of Saturn's magnetic field based on all available data, J. Geophys. Res., 95, 15,257-15,261, doi:10.1029/JA095iA09p15257.

Fuller-Rowell, T. J., and D. S. Evans (1987), Height-integrated Pedersen and Hall conductivity patterns inferred from the TIROS-NOAA satellite data, J. Geophys. Res., 92, 7606-7618.

Galand, M., L. Moore, B. Charnay, I. Müller-Wodarg, and M. Mendillo (2009), Solar primary and secondary ionization at Saturn, J. Geophys. Res., 114, A06313, doi:10.1029/2008JA013981.

Gérard, J.-C., and V. Singh (1982), A model of energy deposition of energetic electrons and EUV emission in the Jovian and Saturnian atmospheres and implications, J. Geophys. Res., 87, 4525-4532. 
Gérard, J.-C., D. Grodent, J. Gustin, A. Saglam, J. T. Clarke, and J. T. Trauger (2004), Characteristics of Saturn's FUV aurora observed with the Space Telescope Imaging Spectrograph, J. Geophys. Res., 109, A09207, doi:10.1029/2004JA010513.

Gérard, J.-C., B. Bonfond, J. Gustin, D. Grodent, and J. T. Clarke (2009), Characteristics of electron precipitation derived from FUV observations of Saturn's aurora, paper presented at Magnetospheres of the Outer Planets, Europlanet Res. Infrastructure, Cologne, Germany.

Grodent, D., J. H. Waite Jr., and J.-C. Gérard (2001), A self-consistent model of Jovian auroral thermal structure, J. Geophys. Res., 106, 12,933-12,952, doi:10.1029/2000JA900129.

Grodent, D., J.-C. Gérard, S. W. H. Cowley, E. J. Bunce, and J. T. Clarke (2005), Variable morphology of Saturn's southern ultraviolet aurora, J. Geophys. Res., 110, A07215, doi:10.1029/2004JA010983.

Grodent, D., A. Radioti, B. Bonfond, and J.-C. Gérard (2010), On the origin of Saturn's outer auroral emission, J. Geophys. Res., 115, A08219, doi:10.1029/2009JA014901

Gurnett, D. A., et al. (2005), Radio and plasma wave observations at Saturn from Cassini's approach and first orbit, Science, 307, 1255-1259, doi:10.1126/science.1105356.

Gustin, J., J.-C. Gérard, W. Pryor, P. D. Feldman, D. Grodent, and G. Holsclaw (2009), Characteristics of Saturn's polar atmosphere and auroral electrons derived from HST/STIS, FUSE and Cassini/UVIS spectra, Icarus, 200, 176-187, doi:10.1016/j.icarus.2008.11.013.

Helled, R., G. Schubert, and J. D. Anderson (2009), Jupiter and Saturn rotation periods, Planet. Space Sci., 57, 1467-1473, doi:10.1016/j.pss.2009. 07.008 .

Hinson, D. P, J. D. Twicken, and E. T. Karayel (1998), Jupiter's ionosphere: New results from Voyager 2 radio occultation measurements, J. Geophys. Res., 103, 9505-9520.

Hiraki, Y., and C. Tao (2008), Parameterization of ionization rate by auroral electron precipitation in Jupiter, Ann. Geophys., 26, 77-86.

Huestis, D. (2008), Hydrogen collisions in planetary atmospheres, ionospheres, and magnetospheres, Planet. Space Sci., 56, 1733-1743, doi:10.1016/j.pss.2008.07.012.

Hui, Y., T. E. Cravens, N. Ozak, and D. R. Schultz (2010), What can be learned from the absence of auroral X-ray emission from Saturn?, J. Geophys. Res., 115, A10239, doi:10.1029/2010JA015639.

Jackman, C. M., and S. W. H. Cowley (2006), A model of the plasma flow and current in Saturn's polar ionosphere under conditions of strong Dungey cycle driving, Ann. Geophys., 24, 1029-1055.

Kelley, M. C. (1989), The Earth's Ionosphere: Plasma Physics and Electrodynamics, Int. Geophys. Ser., vol. 43, Academic, San Diego, Calif.

Kliore, A. J., A. F. Nagy, E. A. Marouf, A. Anabtawi, E. Barbinis, D. U. Fleischman, and D. S. Kahane (2009), Midlatitude and high-latitude electron density profiles in the ionosphere of Saturn obtained by Cassini radio occultation observations, J. Geophys. Res., 114, A04315, doi:10.1029/2008JA013900

Kurth, W. S., et al. (2009), Auroral processes, in Saturn From CassiniHuygens, edited by M. Dougherty, L. W. Esposito, and S. M. Krimigis, chap. 12 , pp. 333-374, Springer, New York, doi:10.1007/978-1-40209217-6.

Lamy, L., B. Cecconi, R. Prangé, P. Zarka, J. D. Nichols, and J. T. Clarke (2009), An auroral oval at the footprint of Saturn's kilometric radio sources, colocated with the UV aurorae, J. Geophys. Res., 114, A10212, doi:10.1029/2009JA014401.

Lamy, L., et al. (2010), Properties of Saturn kilometric radiation measured within its source region, Geophys. Res. Lett., 37, L12104, doi:10.1029/ 2010GL043415.

Lindal, G. F., D. N. Sweetnam, and V. R. Eshleman (1985), The atmosphere of Saturn: An analysis of the Voyager radio occultation measurements, Astron. J., 90, 1136-1146.

Majeed, T., J. C. McConnell, and R. V. Yelle (1991), Vibrationally excited $\mathrm{H}_{2}$ in the outer planets thermosphere: Florescence in the Lyman and Werner bands, Planet. Space Sci., 39, 1591-1606.

Melin, H., S. Miller, T. Stallard, L. M. Trafton, and T. R. Geballe (2007), Variability in the $\mathrm{H}_{3}^{+}$emission of Saturn: Consequences for ionisation rates and temperature, Icarus, 186, 234-241, doi:10.1016/j.icarus.2006. 08.014 .

Menager, H., M. Barthélemy, and J. Lilensten (2010), H Lyman $\alpha$ line in Jovian aurorae: Electron transport and radiative transfer coupled modelling, Astron. Astrophys., 509, 56-65, doi:10.1051/0004-6361/ 200912952

Miller, S., J. Tennyson, and B. T. Sutcliffe (1990), Forbidden rotational and rovibrational transitions in $\mathrm{H} 3+$ : First principles calculations, $J$. Mol. Spectrosc., 141, 104-117, doi:10.1016/0022-2852(90)90281-T.

Miller, S., T. Stallard, H. Melin, and J. Tennyson (2010), H3+ cooling in planetary atmospheres, Faraday Discuss., 147, 283-291, doi:10.1039/ c004152c
Millward, G., S. Miller, T. Stallard, A. D. Aylward, and N. Achilleos (2002), On the dynamics of the Jovian ionosphere and thermosphere: III. The modelling of auroral conductivity, Icarus, 160, 95-107, doi:10.1006/icar.2002.6951

Moore, L., and M. Mendillo (2007), Are plasma depletions in Saturn's ionosphere a signature of time-dependent water input?, Geophys. Res. Lett. 34, L12202, doi:10.1029/2007GL029381.

Moore, L., A. F. Nagy, A. J. Kliore, I. Mueller-Wodarg, J. D. Richardson, and M. Mendillo (2006), Cassini radio occultations of Saturn's ionosphere: Model comparisons using a constant water flux, Geophys. Res. Lett., 33, L22202, doi:10.1029/2006GL027375.

Moore, L., M. Galand, I. Müller-Wodarg, R. Yelle, and M. Mendillo (2008), Plasma temperatures in Saturn's ionosphere, J. Geophys. Res., 113, A10306, doi:10.1029/2008JA013373.

Moore, L., M. Galand, I. Müller-Wodarg, and M. Mendillo (2009), Response of Saturn's ionosphere to solar radiation: Testing parameterizations for thermal electron heating and secondary ionization processes, Planet. Space Sci., 57, 1699-1705, doi:10.1016/j.pss.2009.05.001.

Moore, L., I. Müller-Wodarg, M. Galand, A. Kliore, and M. Mendillo (2010), Latitudinal variations in Saturn's ionosphere: Cassini measurements and model comparisons, J. Geophys. Res., 115, A11317, doi:10.1029/2010JA015692.

Moore, L. E., M. Mendillo, I. C. F. Mueller-Wodarg, and D. L. Murr (2004), Modeling of global variations and ring shadowing in Saturn's ionosphere, Icarus, 172, 503-520, doi:10.1016/j.icarus.2004.07.007.

Moses, J. I., and S. F. Bass (2000), The effects of external material on the chemistry and structure of Saturn's ionosphere, J. Geophys. Res., 105, 7013-7052, doi:10.1029/1999JE001172.

Moses, J. I., B. Bezard, E. Lellouch, G. R. Gladstone, H. Feuchtgruber, and M. Allen (2000), Photochemistry of Saturn's atmosphere: I. Hydrocarbon chemistry and comparisons with ISO observations, Icarus, 143, 244-298, doi:10.1006/icar.1999.6270

Müller-Wodarg, I. C. F., M. Mendillo, R. V. Yelle, and A. D. Aylward (2006), A global circulation model of Saturn's thermosphere, Icarus, 180, 147-160, doi:10.1016/j.icarus.2005.09.002.

Nichols, J. D., et al. (2009), Saturn's equinoctial auroras, Geophys. Res. Lett., 36, L24102, doi:10.1029/2009GL041491.

Perry, J. J., Y. H. Kim, J. L. Fox, and H. S. Porter (1999), Chemistry of the Jovian auroral ionosphere, J. Geophys. Res., 104, 16,541-16,565.

Prangé, R., L. Pallier, K. C. Hansen, R. Howard, A. Vourlidas, R. Courtin, and C. Parkinson (2004), An interplanetary shock traced by planetary auroral storms from the Sun to Saturn, Nature, 432, 78-81, doi:10.1038/ nature02986.

Pryor, W. R., et al. (2009), Saturn auroral images and movies from Cassini UVIS, paper presented at Magnetospheres of the Outer Planets, Europlanet Res. Infrastructure, Cologne, Germany.

Read, P. L., T. E. Dowling, and G. Schubert (2009), Saturn's rotation period from its atmospheric planetary-wave configuration, Nature, 460 608-610, doi:10.1038/nature08194.

Régo, D., R. Prangé, and J.-C. Gérard (1994), Auroral Lyman alpha and $\mathrm{H}_{2}$ bands from the giant planets: 1 . Excitation by proton precipitation in the Jovian atmosphere, J. Geophys. Res., 99, 17,075-17,094, doi:10.1029/ 93JE03432.

Richmond, A. D. (1995), Ionospheric electrodynamics, in Handbook of Atmospheric Electrodynamics, vol. 2, edited by H. Volland, pp. 249-290, CRC Press, Boca Raton, Fla.

Rishbeth, H., and O. K. Garriott (1969), Introduction to Ionospheric Physics, Int. Geophys. Ser., Academic, New York.

Sandel, B. R., D. E. Shemansky, A. L. Broadfoot, J. B. Holberg, and G. R. Smith (1982), Extreme ultraviolet observations from the Voyager 2 encounter with Saturn, Science, 215, 548-553.

Schunk, R. W., and A. F. Nagy (2009), Ionospheres: Physics, Plasma physics, and Chemistry, Atmos. Space Sci. Ser., 2nd ed., 628 pp., Cambridge Univ. Press, Cambridge, U. K.

Smith, G. R., D. E. Shemansky, J. B. Holberg, A. L. Broadfoot, and B. R. Sandel (1983), Saturn's upper atmosphere from the Voyager 2 EUV solar and stellar occultations, J. Geophys. Res., 88, 8667-8678.

Stallard, T., S. Miller, M. Lystrup, N. Achilleos, C. Arridge, and M. Dougherty (2008a), Dusk-brightening event in Saturn's $\mathrm{H}_{3}^{+}$aurora, Astrophys. J., 673, L203-L206.

Stallard, T., S. Miller, H. Melin, M. Lystrup, S. W. H. Cowley, E. J. Bunce, N. Achilleos, and M. Dougherty (2008b), Jovian-like aurorae on Saturn, Nature, 453, 1083-1085, doi:10.1038/nature07077.

Stallard, T., et al. (2008c), Complex structure within Saturn's infrared aurora, Nature, 456, 214-217, doi:10.1038/nature07440.

Stallard, T., H. Melin, S. W. H. Cowley, S. Miller, and M. B. Lystrup (2010), Location and magnetospheric mapping of Saturn's mid-latitude infrared auroral oval, Astrophys. J., 722, L85-L89, doi:10.1088/2041$8205 / 722 / 1 / \mathrm{L} 85$. 
Waite, J. H., Jr. (1981), The ionosphere of Saturn, diploma thesis, Univ. of Mich., Ann Arbor.

Waite, J. H., Jr., T. E. Cravens, J. Kozyra, A. F. Nagy, S. K. Atreya, and R. H. Chen (1983), Electron precipitation and related aeronomy of the Jovian thermosphere and ionosphere, J. Geophys. Res., 88, 6143-6163.

Woods, T. N. (2008), Recent advances in observations and modeling of the solar ultraviolet and X-ray spectral irradiance, $A d v$. Sp. Res., 42, 895-902, doi:10.1016/j.asr.2007.09.026.

Woods, T. N., et al. (2000), TIMED Solar EUV Experiment, Phys. Chem. Earth, Part C, 25, 393-396.

Woods, T. N., F. G. Eparvier, S. M. Bailey, P. C. Chamberlin, J. Lean, G. J. Rottman, S. C. Solomon, W. K. Tobiska, and D. L. Woodraska (2005), Solar EUV Experiment (SEE): Mission overview and first results, J. Geophys. Res., 110, A01312, doi:10.1029/2004JA010765.
Yelle, R. V., and S. Miller (2004), Jupiter's thermosphere and ionosphere, in Jupiter: The Planet, Satellites, and Magnetospheres, edited by F. Bagenal et al., chap. 12, pp. 185-218, Cambridge Univ. Press, New York.

Zarka, P., L. Lamy, B. Cecconi, R. Prangé, and H. O. Rucker (2007), Modulation of Saturn's radio clock by solar wind speed, Nature, 450, 265-267, doi:10.1038/nature06237.

M. Galand and I. Mueller-Wodarg, Space and Atmospheric Physics Group, Department of Physics, Imperial College London, Prince Consort Road, London SW7 2AZ, UK. (m.galand@imperial.ac.uk)

M. Mendillo and L. Moore, Center for Space Physics, Boston University, 725 Commonwealth Ave., Boston, MA 02215, USA.

S. Miller, Atmospheric Physics Laboratory, Department of Physics and Astronomy, University College London, London WC1 6BT, UK. 